\title{
Nonlinear Dynamic Analysis and Synchronization of Four-Dimensional Lorenz-Stenflo System and Its Circuit Experimental Implementation
}

\author{
Cheng-Hsiung Yang and Cheng-Lin Wu \\ Graduate Institute of Automation and Control, National Taiwan University of Science and Technology, No. 43, Section 4, \\ Keelung Road, Taipei 106, Taiwan
}

Correspondence should be addressed to Cheng-Hsiung Yang; chyang123123@mail.ntust.edu.tw

Received 2 April 2014; Accepted 25 May 2014; Published 7 August 2014

Academic Editor: Xiao He

Copyright (C) 2014 C.-H. Yang and C.-L. Wu. This is an open access article distributed under the Creative Commons Attribution License, which permits unrestricted use, distribution, and reproduction in any medium, provided the original work is properly cited.

Recently many chaotic systems' circuits are designed to generate phenomenon of chaos signals. The ability to synchronize chaotic circuits opens a great number of ways to use them in application signals masking. In this paper, first a new nonlinear chaotic dynamical system had be design, analyze and build circuit. Second, using GYC, partial region stability theory is applied to adaptive control for two identical chaotic systems with uncertain parameters. The results of numerical simulation are performed to verify examples of the proposed nonlinear controllers.

\section{Introduction}

Chaotic systems provide a rich mechanism for signal design and generation, with potential applications to communications and signal processing. Because chaotic signals are typically broadband, noise-like, and difficult to predict, they can be used in various contexts for masking information bearing waveforms. They can also be used as modulating waveforms in spread spectrum systems.

This can be useful in many practical circumstances like securing communication channels [1], masking signals [2], spreading data sequence, or for generating random signals [3]. Of course, chaos is an unwanted phenomenon in many situations. The essential problem which is faced here is how to distinguish such motion from long transient behavior. A spectrum called Lyapunov exponents (LE) is often used as a quantifier [4] of chaotic motion or regular motion. These real numbers measure the average ratio of the exponential separation of the two neighborhood trajectories. For chaos it is necessary to have at least one positive of Lyapunov exponents; the second one represents a direction of the flow and must converge to zero. The last one must be negative with the largest absolute value, since the flow is dissipative. Circuits of nonlinear dynamic system provide an excellent tool for the study of chaotic behavior. Some of these circuits treat time as a discrete variable, employing sample-and-hold subcircuits and analog multipliers to model iterated maps such as the logistic map $[5,6]$.

In this paper, an adaptive generalized synchronization of the four-dimensional Lorenz-Stenflo system [7-12] with uncertain chaotic parameters strategy by GYC partial region stability theory is proposed [13-16]. By using the GYC partial region stability theory the Lyapunov function is a simple linear homogeneous function of states and the structure of controllers are simpler and have less simulation error because they are in lower order than that of traditional controllers design.

This paper is organized as follows. In Section 2, the four-dimensional Lorenz-Stenflo system analyzes characteristics of nonlinear dynamic behavior, construction realization circuit of the four-dimensional Lorenz-Stenflo system. In Section 3, adaptive generalized chaos synchronization with uncertain parameters strategy by GCY partial region stability theory is proposed. In Section 4, numerical results of adaptive generalized synchronization of the four-dimensional LorenzStenflo system with uncertain chaotic parameters using GYC 


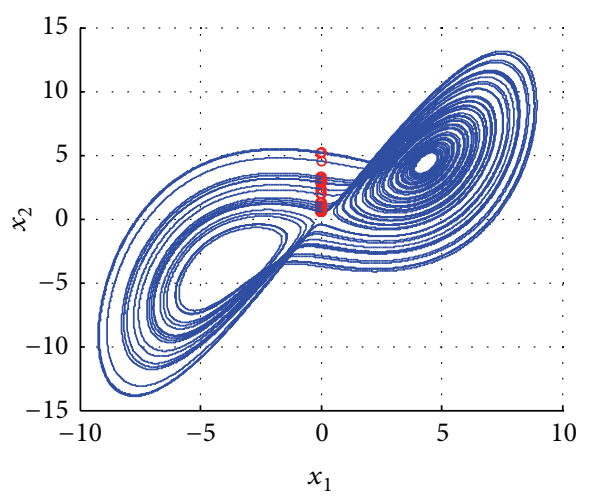

(a)

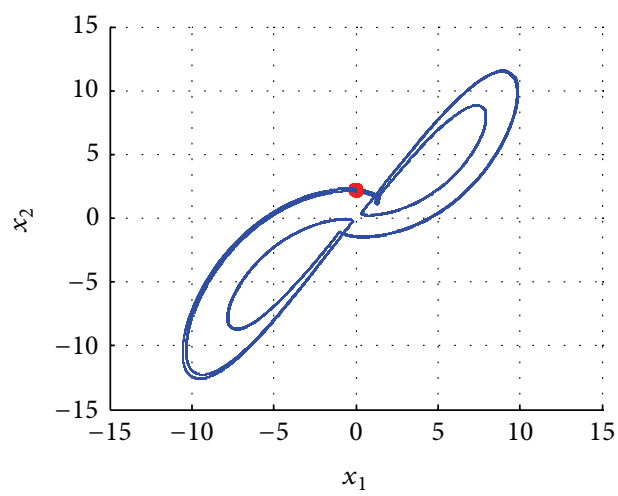

(c)

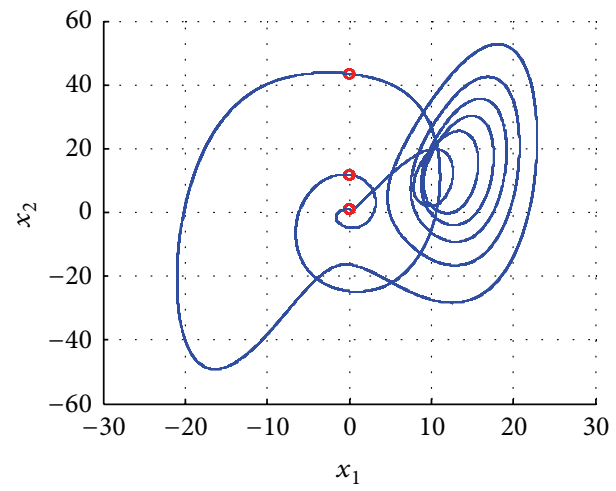

(e)

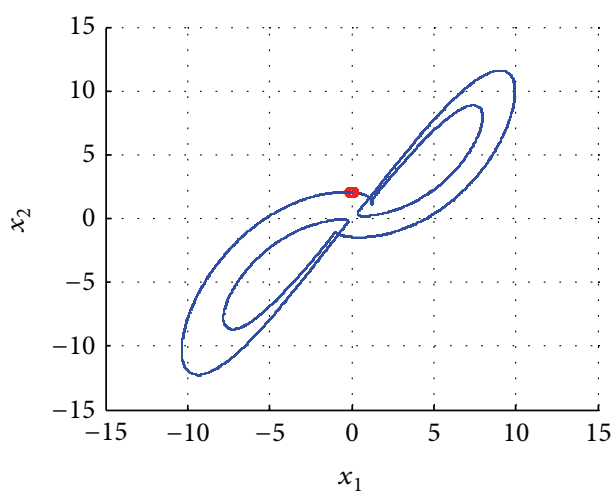

(b)

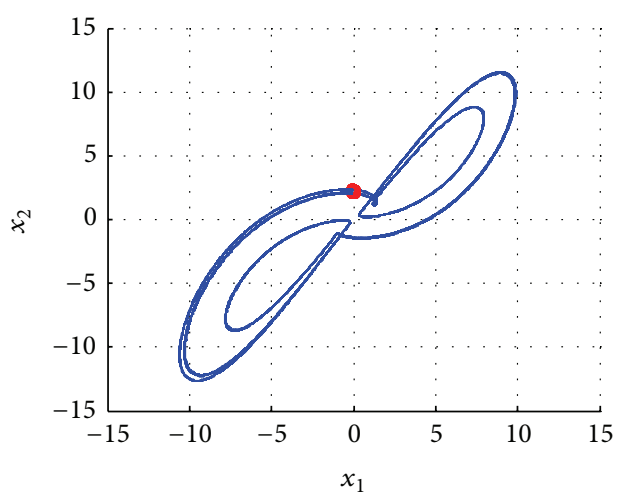

(d)

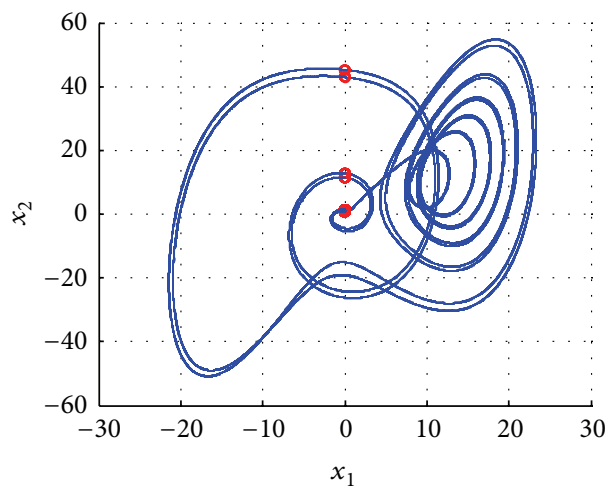

(f)

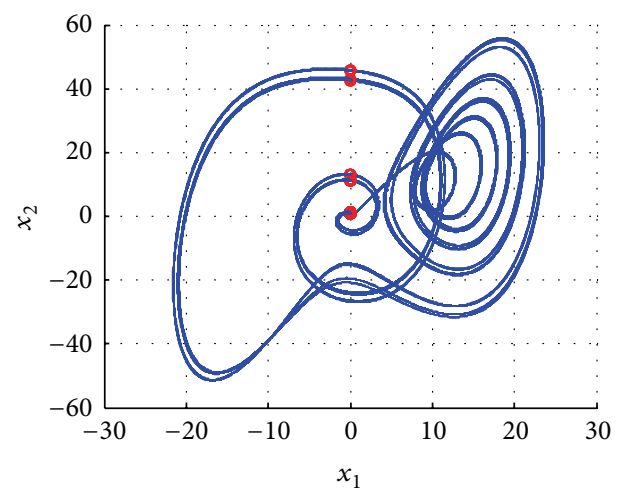

(g)

Figure 1: Phase portraits and Poincaré maps for (a) chaotic, (b) period-1, (c) period-2, (d) period-4, (e) period-3, (f) period-6, and (g) period-12, respectively, of the four-dimensional Lorenz-Stenflo system. 


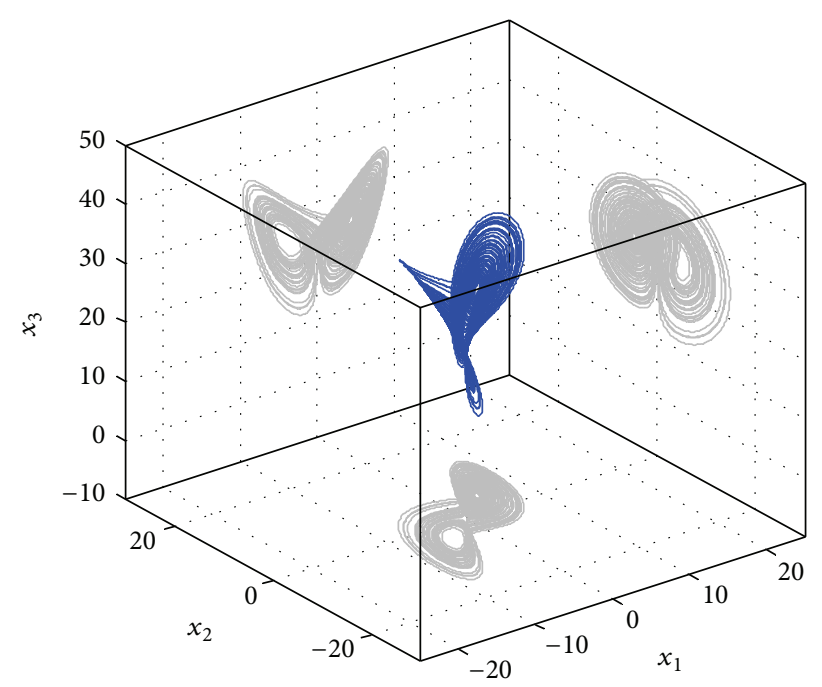

(a)

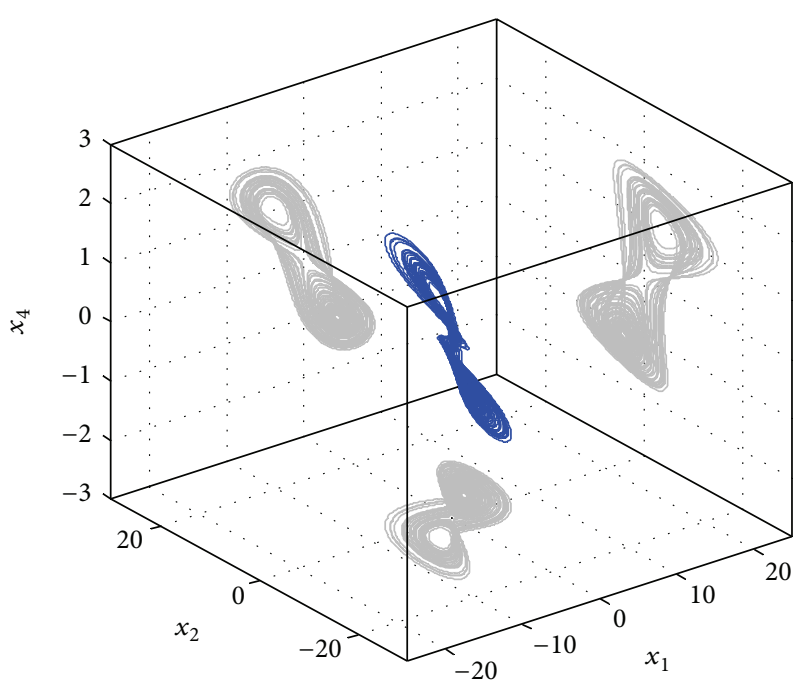

(b)

FIGURE 2: Three dimensions phase portrait of the four-dimensional Lorenz-Stenflo system and its projection.

partial region stability. Finally, some concluding remarks are given in Section 5.

\section{Chaotic Dynamic Analysis of a Four- Dimensional Lorenz-Stenflo System}

2.1. Phase Portraits and Poincaré Map. The Lorenz-Stenflo system is described as $[7,8]$

$$
\begin{aligned}
& \dot{x}_{1}=a x_{2}-a x_{1}+b x_{4} \\
& \dot{x}_{2}=c x_{1}-x_{1} x_{3}-x_{2} \\
& \dot{x}_{3}=x_{1} x_{2}-d x_{3} \\
& \dot{x}_{4}=-x_{1}-a x_{4},
\end{aligned}
$$

where $a$ is Prandtl number, $b$ is rotation number, $c$ is Rayleigh number, and $d$ is geometric parameter.
The $b x_{4}$ of Lorenz-Stenflo system from first item changes to third item, new four-dimensional Lorenz-Stenflo system.

Consider

$$
\begin{aligned}
& \dot{x}_{1}=a x_{2}-a x_{1} \\
& \dot{x}_{2}=c x_{1}-x_{1} x_{3}-x_{2} \\
& \dot{x}_{3}=x_{1} x_{2}-d x_{3}+b x_{4} \\
& \dot{x}_{4}=-x_{1}-a x_{4} .
\end{aligned}
$$

The Lorenz-Stenflo system has many dynamical behaviors that are one chaotic motion and six different periodic motions as shown in Figure 1. To have a better understanding of the four-dimensional Lorenz-Stenflo system, we use an innovative technique to present the three-dimensional phase portraits shown in Figure 2. These figures plot three dimensions phase portraits and their projection simultaneously. 


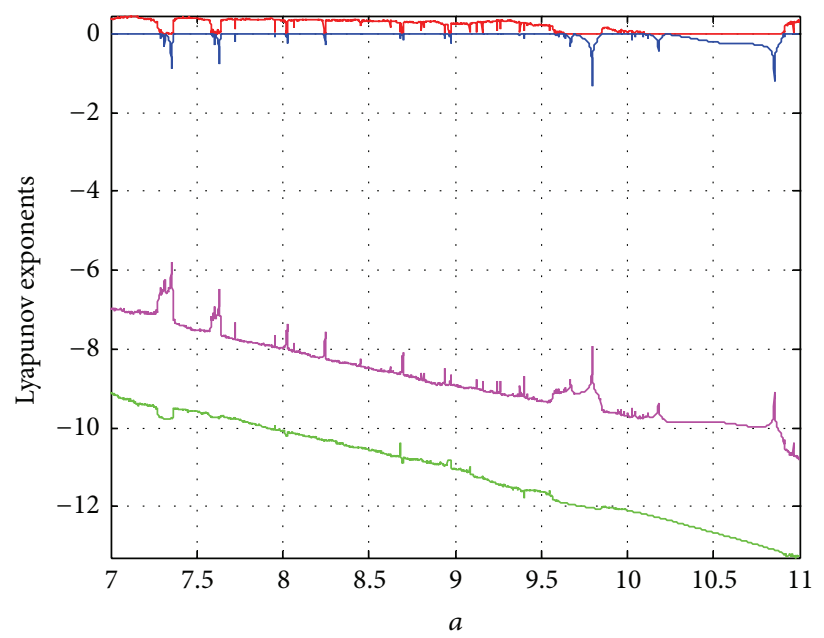

(a)

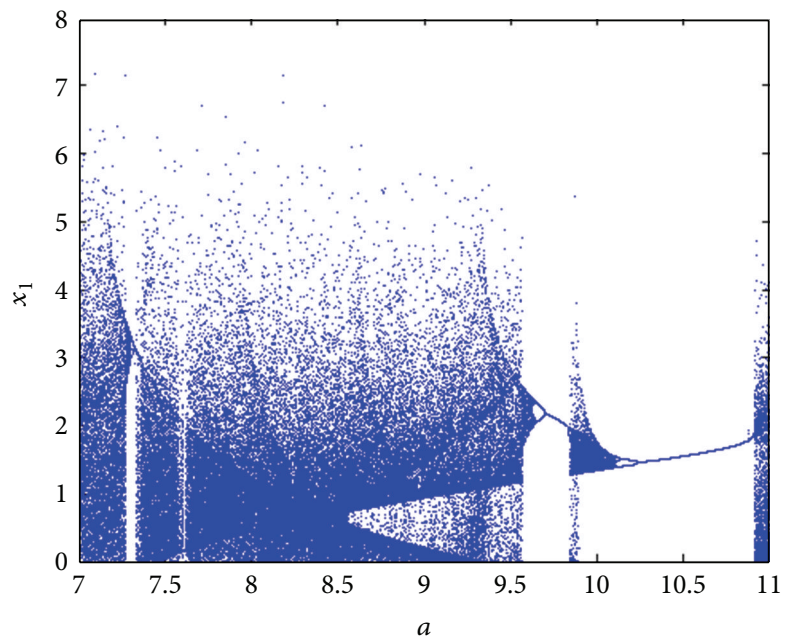

(b)

FIGURE 3: Lyapunov exponents and bifurcation diagrams of the four-dimensional Lorenz-Stenflo system with parameters $b=1.5, c=26$, and $d=0.7$.

Thus, we can comprehend the four-dimensional LorenzStenflo system much easier.

2.2. Equilibrium Analysis. The equilibrium points of the system are shown as follows:

$$
\begin{aligned}
& E_{0}(0,0,0,0) \\
& E_{1}\left(\begin{array}{l}
\frac{b-\sqrt{b^{2}-4 a^{2} d+4 a^{2} c d}}{2 a}, \frac{b-\sqrt{b^{2}-4 a^{2} d+4 a^{2} c d}}{2 a}, c-1, \\
\frac{-b+\sqrt{b^{2}-4 a^{2} d+4 a^{2} c d}}{2 a^{2}}
\end{array}\right) \\
& E_{2}\left(\begin{array}{l}
\frac{b+\sqrt{b^{2}-4 a^{2} d+4 a^{2} c d}}{2 a}, \frac{b+\sqrt{b^{2}-4 a^{2} d+4 a^{2} c d}}{2 a}, c-1, \\
\frac{b+\sqrt{b^{2}-4 a^{2} d+4 a^{2} c d}}{2 a^{2}}
\end{array}\right) .
\end{aligned}
$$

The parameters of four-dimensional Lorenz-Stenflo system are $a=3.7, b=1.5, c=26$, and $d=0.7$, so the equilibrium points become

$$
\begin{aligned}
& E_{0}(0,0,0,0) \\
& E_{1}(-3.98551,-3.98551,25,1.07716) \\
& E_{2}(4.39091,4.39091,25,-1.18673) .
\end{aligned}
$$

For the equilibrium point $E_{0}$, the Jacobian matrix of the system is

$$
J_{0}=\left[\begin{array}{cccc}
-a & a & 0 & 0 \\
c & -1 & 0 & 0 \\
0 & 0 & -d & b \\
-1 & 0 & 0 & -a
\end{array}\right]=\left[\begin{array}{cccc}
-3.7 & 3.7 & 0 & 0 \\
26 & -1 & 0 & 0 \\
0 & 0 & -0.7 & 1.5 \\
-1 & 0 & 0 & -3.7
\end{array}\right]
$$




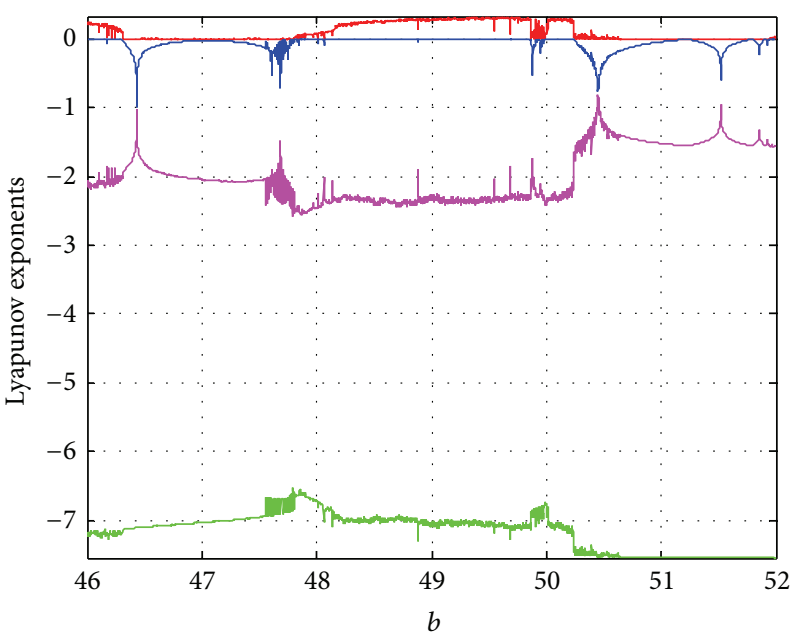

(a)

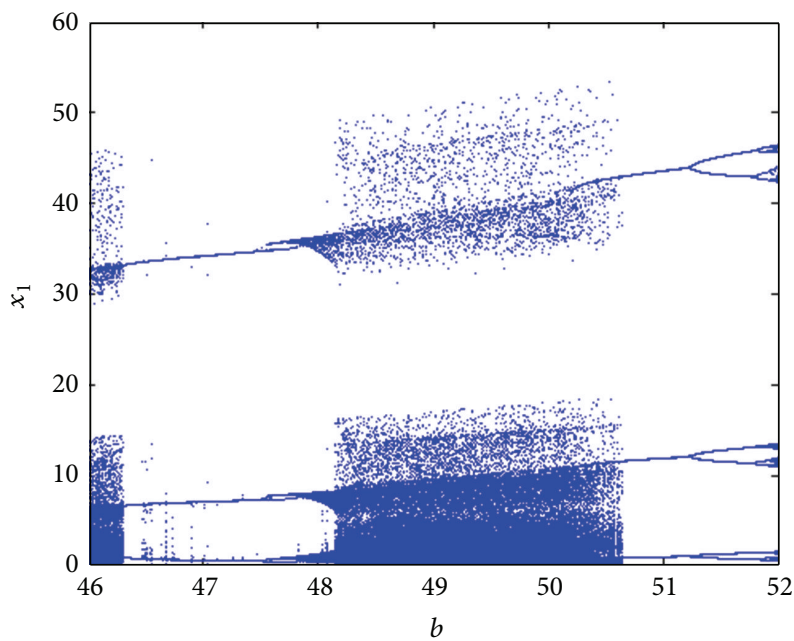

(b)

FIGURE 4: Lyapunov exponents and bifurcation diagrams of the four-dimensional Lorenz-Stenflo system with parameters $a=3.7, c=26$, and $d=0.7$.

The eigenvalues of the Jacobian matrix below are obvious:

$$
\begin{array}{cc}
\lambda_{1}=3.7, & \lambda_{2}=-1, \\
\lambda_{3}=-0.7, & \lambda_{4}=-3.7 .
\end{array}
$$

Then we linearize the system about the nonzero equilibrium points and obtaine the Jacobian matrix:

$$
J_{n z}=\left[\begin{array}{cccc}
-a & a & 0 & 0 \\
c-x_{3} & -1 & -x_{1} & 0 \\
x_{2} & x_{1} & -d & b \\
-1 & 0 & 0 & -a
\end{array}\right] .
$$

The $J_{n z}$ eigenvalues are

$$
\begin{aligned}
\operatorname{det}\left|J_{n z}-\lambda I\right| & =\left[\begin{array}{cccc}
-a-\lambda & a & 0 & 0 \\
c-x_{3} & -1-\lambda & -x_{1} & 0 \\
x_{2} & x_{1} & -d-\lambda & b \\
-1 & 0 & 0 & -a-\lambda
\end{array}\right] \\
& =\lambda^{4}+(2 a+d+1) \lambda^{3}
\end{aligned}
$$

$$
\begin{aligned}
& +\left(a\left(2+2 d+x_{3}-c\right)+a^{2}+d+x_{1}^{2}\right) \lambda^{2} \\
& +\left(a^{2}+2 a d-a^{2} c+a^{2} d-a c d\right. \\
& \left.\quad+2 a x_{1}^{2}+a^{2} x_{3}+a b x_{3}+a x_{1} x_{2}\right) \lambda \\
& +a^{2}\left(x_{1}^{2}+x_{1} x_{2}+d x_{3}\right)-a b x_{1} \\
& +a^{2} d-a^{2} c d .
\end{aligned}
$$

By letting $\operatorname{det}\left|J_{n z}-\lambda I\right|=0$, the eigenvalues of the matrix at each equilibrium point can be obtained as shown in Table 1. The eigenvalues of each equilibrium point are the same. And all the equilibrium points are unstable, since at least one eigenvalue has positive real part for each equilibrium point. 


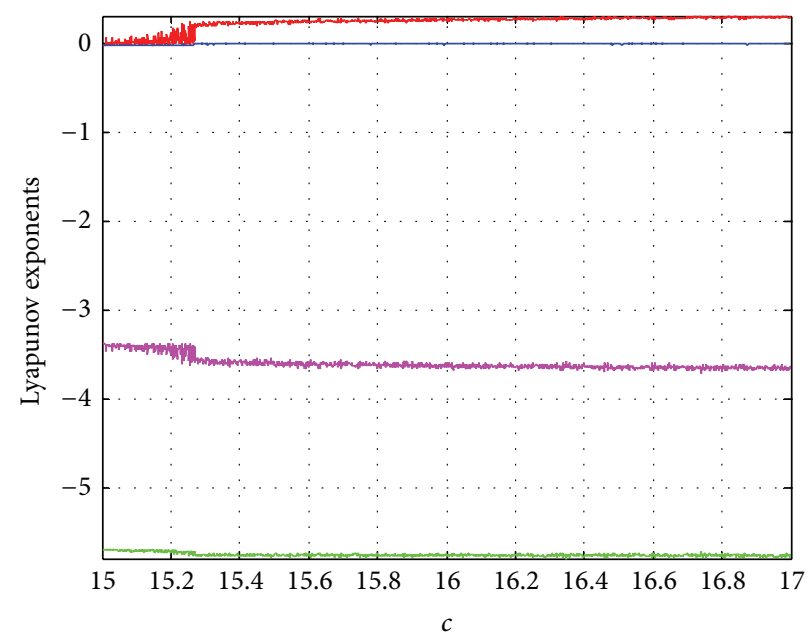

(a)

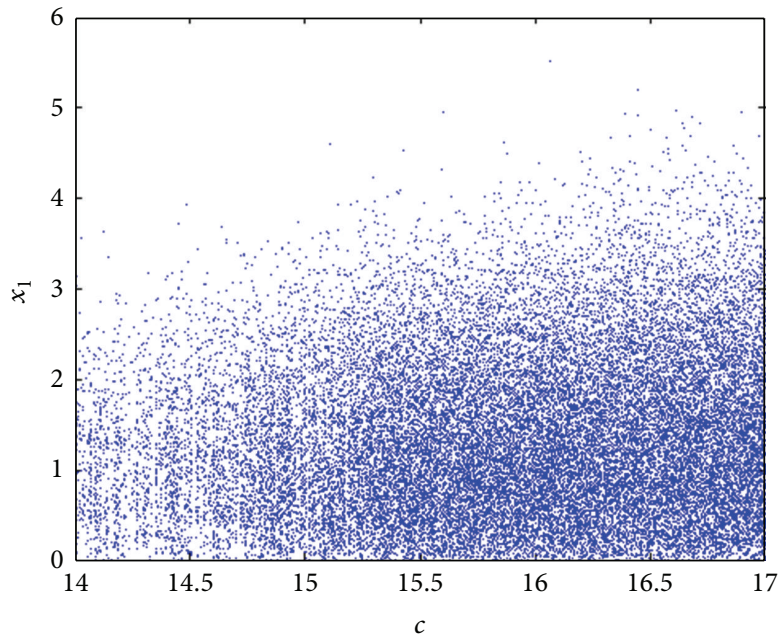

(b)

FIGURE 5: Lyapunov exponents and bifurcation diagrams of the four-dimensional Lorenz-Stenflo system with parameters $a=3.7, b=1.5$, and $d=0.7$.

TABLE 1: Eigenvalues and stability of equilibrium points.

\begin{tabular}{lrr}
\hline Equilibrium point & Eigenvalues & Stable/Unstable \\
\hline$E_{0}$ & $\lambda_{1}=3.7, \lambda_{2}=-1, \lambda_{3}=-0.7, \lambda_{4}=-3.7$ & Unstable \\
$E_{1}$ & $\lambda_{1}=2.152-6.27 i, \lambda_{2}=-12.5777, \lambda_{3}=2.152+6.27 i, \lambda_{4}=-0.826$ & Unstable \\
$E_{2}$ & $\lambda_{1}=2.1489+6.50198 i, \lambda_{2}=-12.5416, \lambda_{3}=2.1489-6.50198 i, \lambda_{4}=-0.8561$ & Unstable \\
\hline
\end{tabular}

2.3. Divergence Analysis. The divergence represents the volume density of the outward flux of a vector field. The divergence of a system implies whether this system dissipative or not. For the four-dimensional Lorenz-Stenflo system, its divergence is

$$
\operatorname{div} \Phi=\nabla \cdot \Phi=\frac{\partial \Phi}{\partial x_{1}}+\frac{\partial \Phi}{\partial x_{2}}+\frac{\partial \Phi}{\partial x_{3}}+\frac{\partial \Phi}{\partial x_{4}}=-a-1-d-a,
$$

where $\Phi=\left(\dot{x}_{1}, \dot{x}_{2}, \dot{x}_{3}, \dot{x}_{4}\right)$.
The parameters are taken as $a=3.7, b=1.5, c=26$, and $d=0.7$; hence, the divergence of the system becomes

$$
\operatorname{div} \Phi=3.7-1+0.7-3.7=-9.1 \text {. }
$$

So, (2) is dissipative, with an exponential contraction rate:

$$
\frac{d \Phi}{d t}=e^{-(2 a+d+1)}
$$

So, a volume element $\Pi_{0}$ is contracted by the flow into a volume element $\Phi_{0} e^{-(2 a+d+1)}$ in time. All orbits of 


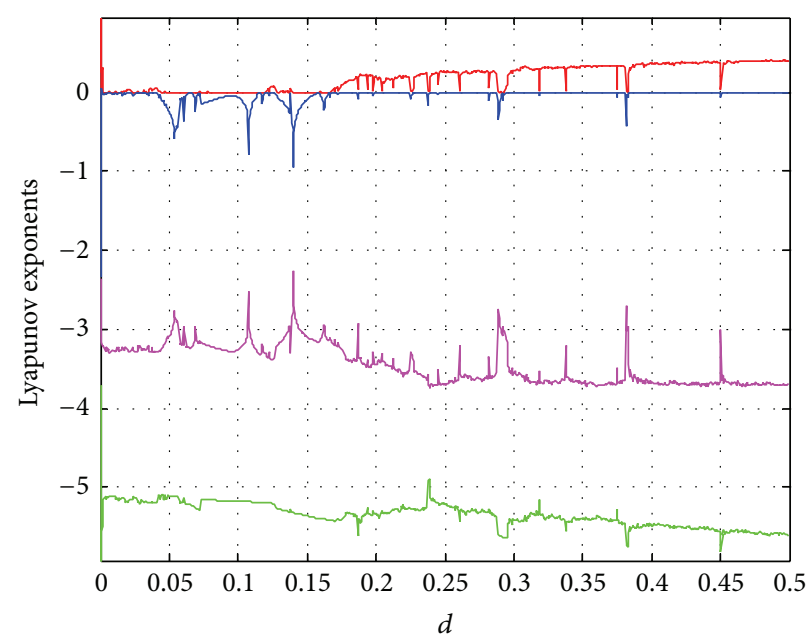

(a)

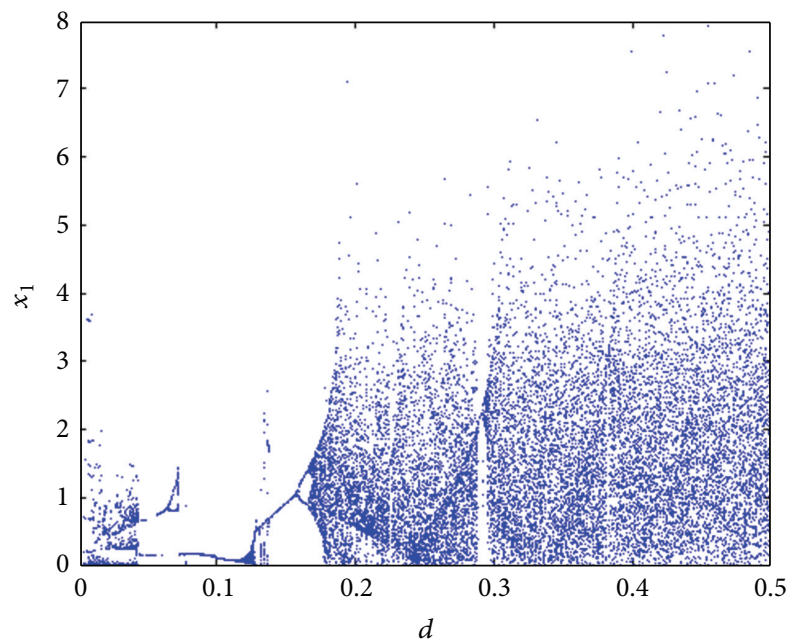

(b)

FIGURE 6: Lyapunov exponents and bifurcation diagrams of the four-dimensional Lorenz-Stenflo system with parameters $a=3.7, b=1.5$, and $c=26$.

(2) are ultimately confined to a set having zero volume and asymptotic motion settles onto an attractor. The fourdimensional Lorenz-Stenflo system has bund system.

2.4. Bifurcation Diagram. For numerical analysis, all of the initial conditions are $x_{1}(0)=20, x_{2}(0)=20, x_{3}(0)=20$, and $x_{4}(0)=20$, in this and next sections. The bifurcation diagrams of the four-dimensional Lorenz-Stenflo system are plotted in Figure 3(a) to Figure 6(a). The parameters $a, b$, $c$, and $d$ in (2) will be varied, respectively, for plotting the bifurcation diagrams. From the bifurcation diagrams, we can learn more about the behaviors of the four-dimensional Lorenz-Stenflo system.

2.5. Lyapunov Exponent and Lyapunov Dimension. The Lyapunov exponent may be used to measure the sensitive dependence on initial conditions. Once the Lyapunov exponent is greater than zero at specific value of parameters, it is said to be chaos, otherwise, periodic solution. The Lyapunov exponents diagrams of the four-dimensional Lorenz-Stenflo system are shown in Figure 3(b) to Figure 6(b). In order to verify the chaotic behavior of the system, Lyapunov exponents diagrams and bifurcation diagrams can be compared together.

The Lyapunov dimension has been proposed by Frederickson et al. [17] to measure the complexity of the attractor. The relation between Lyapunov dimension and Lyapunov exponent is

$$
d_{L}=j+\frac{\sum_{i=1}^{j} \lambda_{i}}{\left|\lambda_{j+1}\right|},
$$

where $\lambda_{i}$ is the largest Lyapunov exponent and $j$ is defined by the condition as

$$
\sum_{i=1}^{j} \lambda_{i} \geq 0, \quad \sum_{i=1}^{j+1} \lambda_{i}<0 .
$$

The Lyapunov dimension diagrams are plotted in Figure 7. 


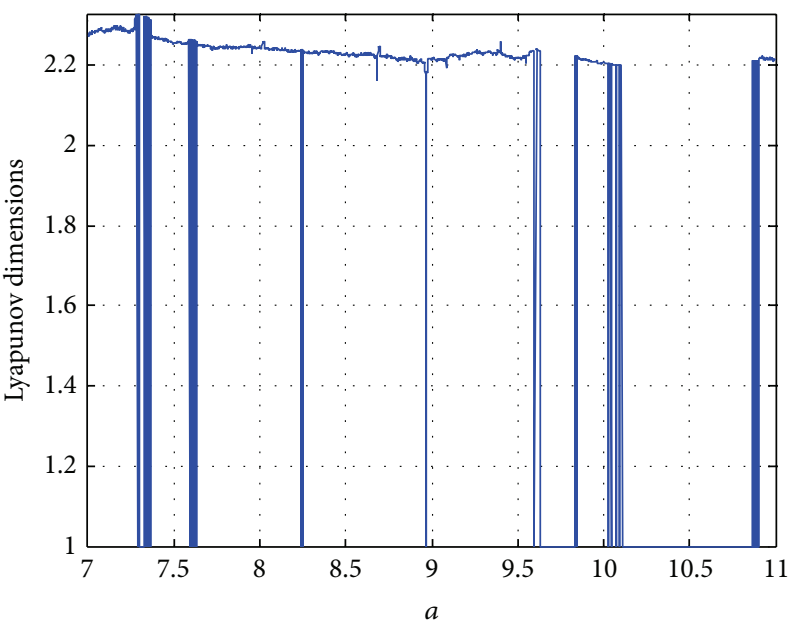

(a)

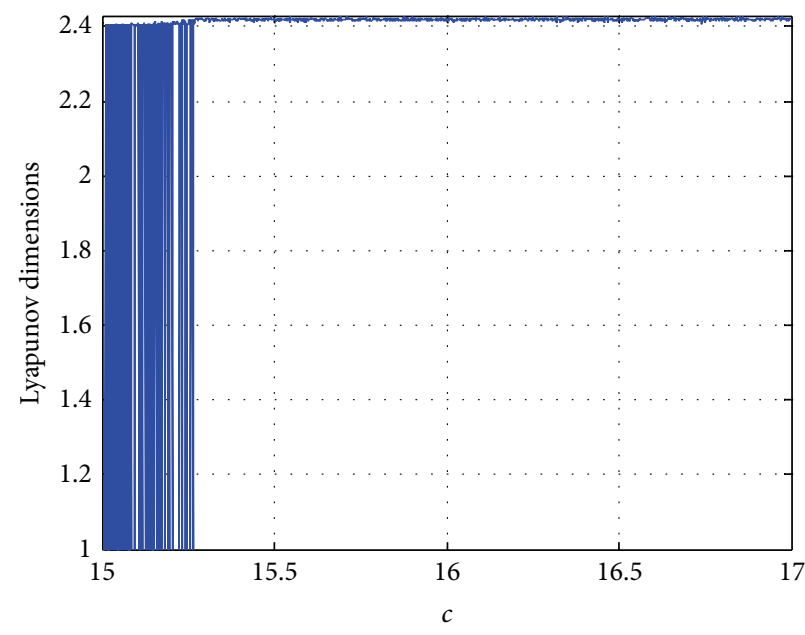

(c)

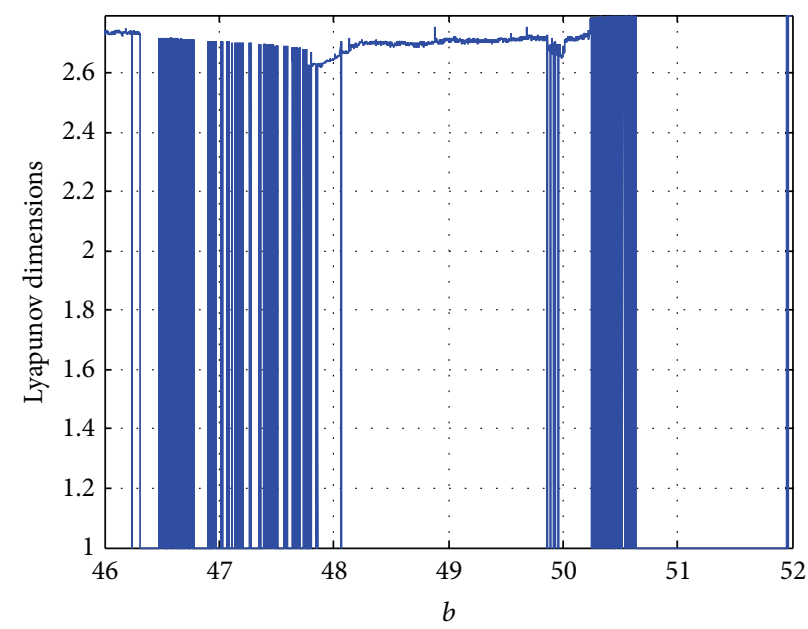

(b)

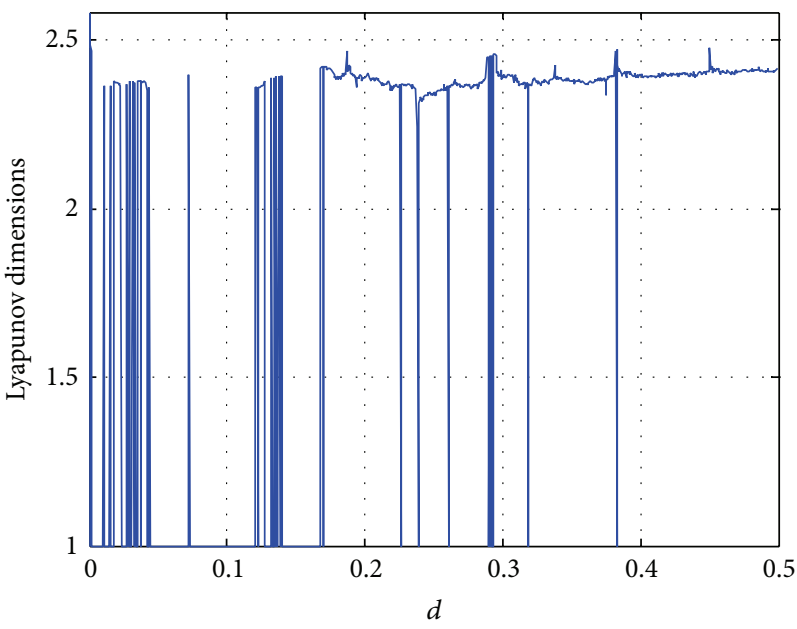

(d)

FIgURE 7: Lyapunov dimension diagrams of the four-dimensional Lorenz-Stenflo system.

2.6. Electronic Circuit Implementation. The four-dimensional Lorenz-Stenflo system can be modeled by an electronic circuit [18-21]. The main electronic components in the circuit are integrated amplifiers and inverting amplifiers. In this circuit simulation, we use electronic circuit simulation software named Multisim. All the parameters and initial conditions are the same as numerical simulation. But here we modify the value of capacitors to decrease the output voltage within a range from -12.0 to 12.0 , and all the state values reduced tenfold in circuit simulation. The chaotic circuit configuration diagram is shown in Figure 8, and the governing integral equation of the circuit can be written as

$$
\begin{aligned}
& \dot{x}_{1}=\frac{1}{R_{1} C_{1}} x_{2}-\frac{1}{R_{2} C_{1}} x_{1}, \\
& \dot{x}_{2}=\frac{1}{R_{3} C_{2}} x_{1}-\frac{1}{R_{4} C_{2}} x_{1} x_{3}-\frac{1}{R_{5} C_{2}} x_{2},
\end{aligned}
$$

$$
\begin{aligned}
& \dot{x}_{3}=\frac{1}{R_{7} C_{3}} x_{1} x_{2}+\frac{1}{R_{6} C_{3}} x_{4}-\frac{1}{R_{8} C_{3}} x_{3}, \\
& \dot{x}_{4}=-\frac{1}{R_{9} C_{4}} x_{1}-\frac{1}{R_{10} C_{4}} x_{4} .
\end{aligned}
$$

Components of the chaotic system circuit are chosen to be $R_{1}=R_{2}=R_{10}=270 \mathrm{k} \Omega, R_{3}=38.461 \mathrm{k} \Omega, R_{6}=667 \mathrm{k} \Omega$, $R_{4}=R_{5}=R_{7}=R_{9}=1 \mathrm{M} \Omega, R_{8}=1429 \mathrm{k} \Omega, R_{11}=R_{12}=$ $R_{13}=R_{14}=R_{15}=R_{16}=R_{17}=R_{18}=100 \mathrm{k} \Omega$, and $C_{1}=C_{2}=C_{3}=C_{4}=1 \mu \mathrm{F}$. The voltage output signals of the chaotic circuit simulated in Multisim software are shown in Figure 9. In addition, the real circuit of the four-dimensional Lorenz-Stenflo system was constructed on a breadboard shown in Figure 13. The apparatus and the components used in the circuit are listed in Table 2. The experimental setup is shown in Figure 11 and the experimental results are shown in Figure 12. 


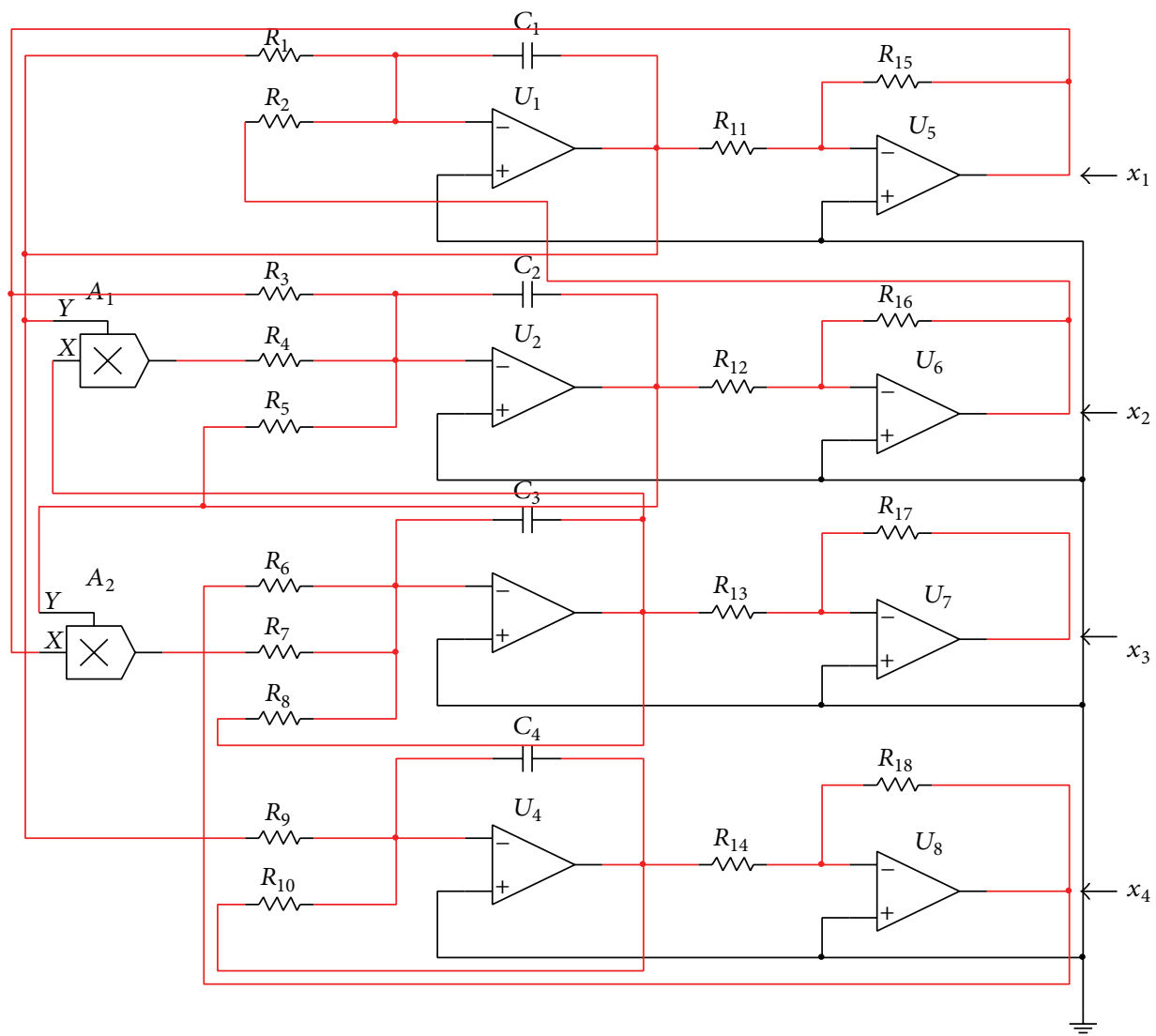

FIGURE 8: Chaotic circuit of the four-dimensional Lorenz-Stenflo system.

In order to have a better concept about chaotic motions and periodic motions, we present a four-dimensional diagram, which is a combination of the phase portraits of the circuits simulation and the bifurcation diagram of variable parameter a, plotted in Figure 10.

\section{Adaptive Generalized Synchronization of the Four-Dimensional Lorenz-Stenflo System with Uncertain Chaotic Parameters via GYC Partial Region Stability Theory Strategy}

Consider the master system

$$
\dot{x}=f(t, x, A(t)),
$$

and the slave system

$$
\dot{y}=f(t, y, \widehat{A}(t))+u
$$

where $x=\left[x_{1}, x_{2}, \ldots, x_{n}\right]^{T} \in R^{n}$ and $y=\left[y_{1}, y_{2}, \ldots, y_{n}\right]^{T} \in$ $R^{n}$ denote the master state vector and slave state vector, respectively, $f$ is nonlinear vector functions, $A(t)$ is uncertain chaotic parameter in $f, \widehat{A}(t)$ is estimates of uncertain chaotic parameter in $f$, and $u=\left[u_{1}, u_{2}, \ldots, u_{n}\right]^{T} \in R^{n}$ is a control input vector.
Our goal is to design a controller $u(t)$ so that the state vector of the slave system (16) asymptotically approaches the state vector of the master system (15) plus a given vector function $F(t)=\left[F_{1}(t), F_{2}(t), \ldots, F_{n}(t)\right]^{T}$ which is either a regular or a chaotic function of time, and finally the synchronization will be accomplished in the sense that the limit of the error vector $e(t)=\left[e_{1}, e_{2}, \ldots, e_{n}\right]^{T}$ approaches zero:

$$
\lim _{t \rightarrow \infty} e(t)=0
$$

where $e_{i}=x_{i}-y_{i}+F_{i}(t)+K e_{i}, i=1,2, \ldots, n$. The $K e_{i}$ are positive constants which make the error dynamics always happens in the first quadrant.

From (17) we have

$$
\dot{e}_{i}=\dot{x}_{i}-\dot{y}_{i}+\dot{F}_{i}(t), \quad i=1,2, \ldots, n .
$$

Introducing (15) and (16) in (18), we get

$$
\dot{e}=f(t, x, A(t))-f(t, y, \widehat{A}(t))+\dot{F}(t)-u(t) .
$$

A Lyapunov function $V(e, \widetilde{A})$ is chosen as a positive definite function in the first quadrant.

Consider

$$
V(e, \widetilde{A})=e+\widetilde{A},
$$




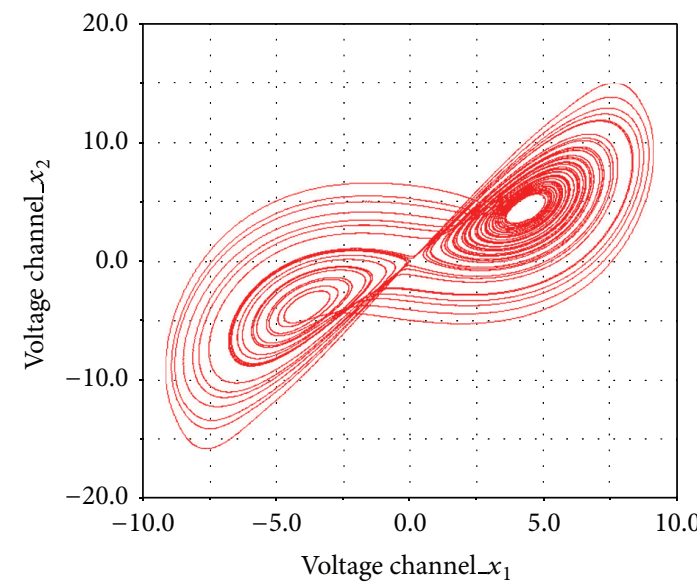

(a)

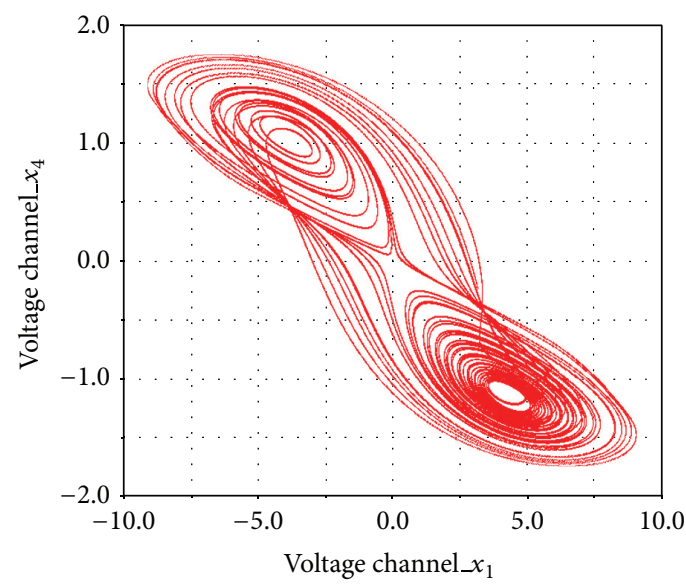

(c)

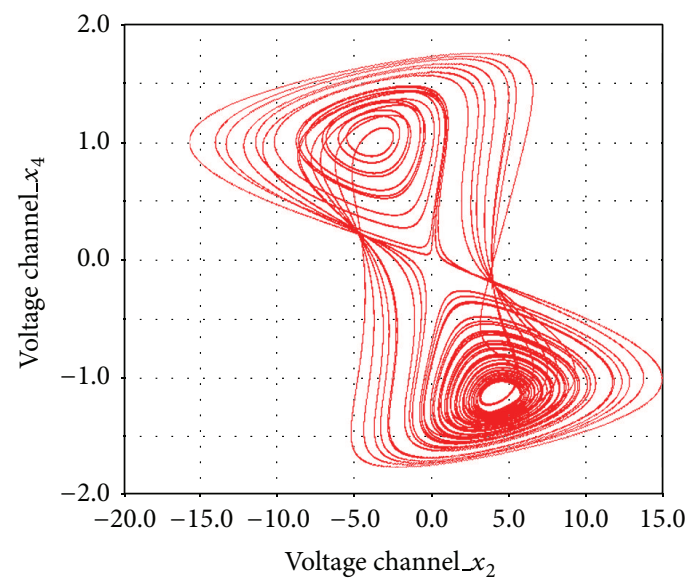

(e)

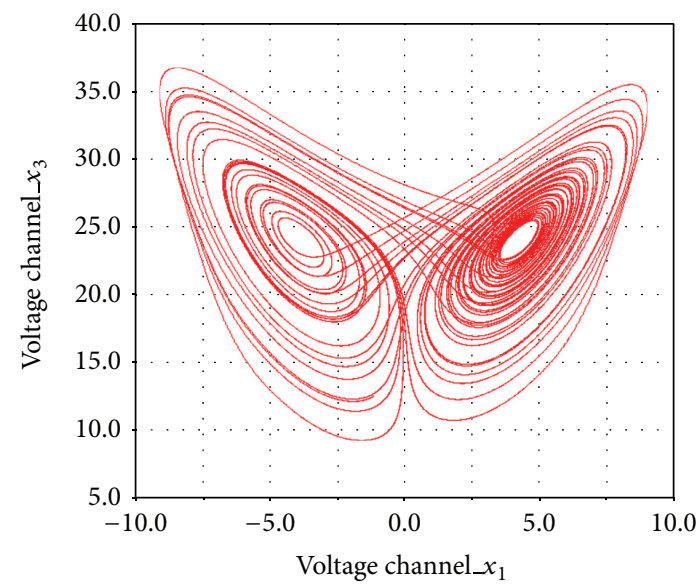

(b)

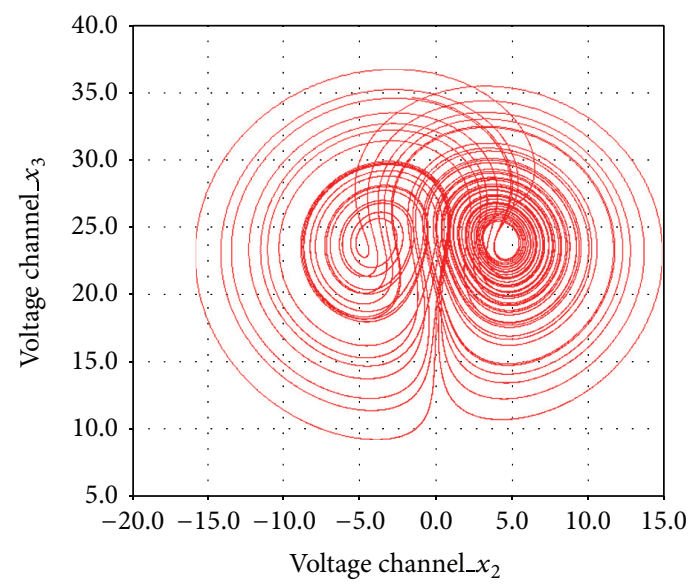

(d)

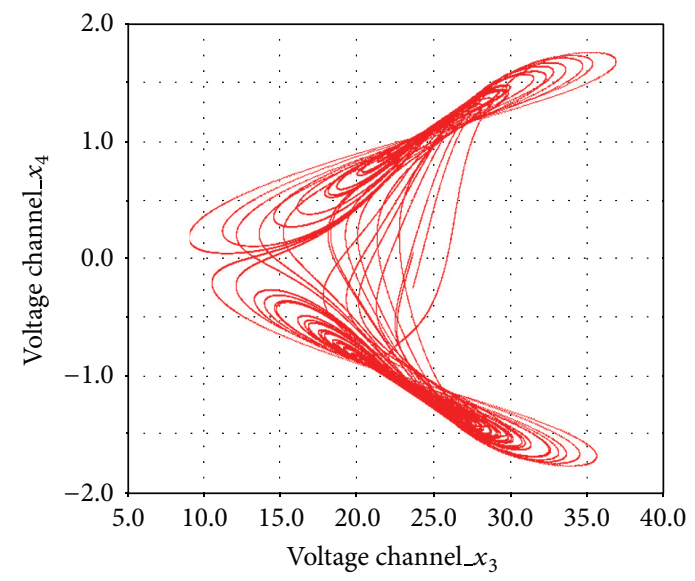

(f)

FIGURE 9: Oscilloscope graphics for voltage signals outputs of the four-dimensional Lorenz-Stenflo system: (a) $V_{x_{1}}-V_{x_{2}}$, (b) $V_{x_{1}}-V_{x_{3}}$, (c) $V_{x_{1}}-V_{x_{4}}$, (d) $V_{x_{2}}-V_{x_{3}}$, (e) $V_{x_{2}}-V_{x_{4}}$, and (f) $V_{x_{3}}-V_{x_{4}}$. 


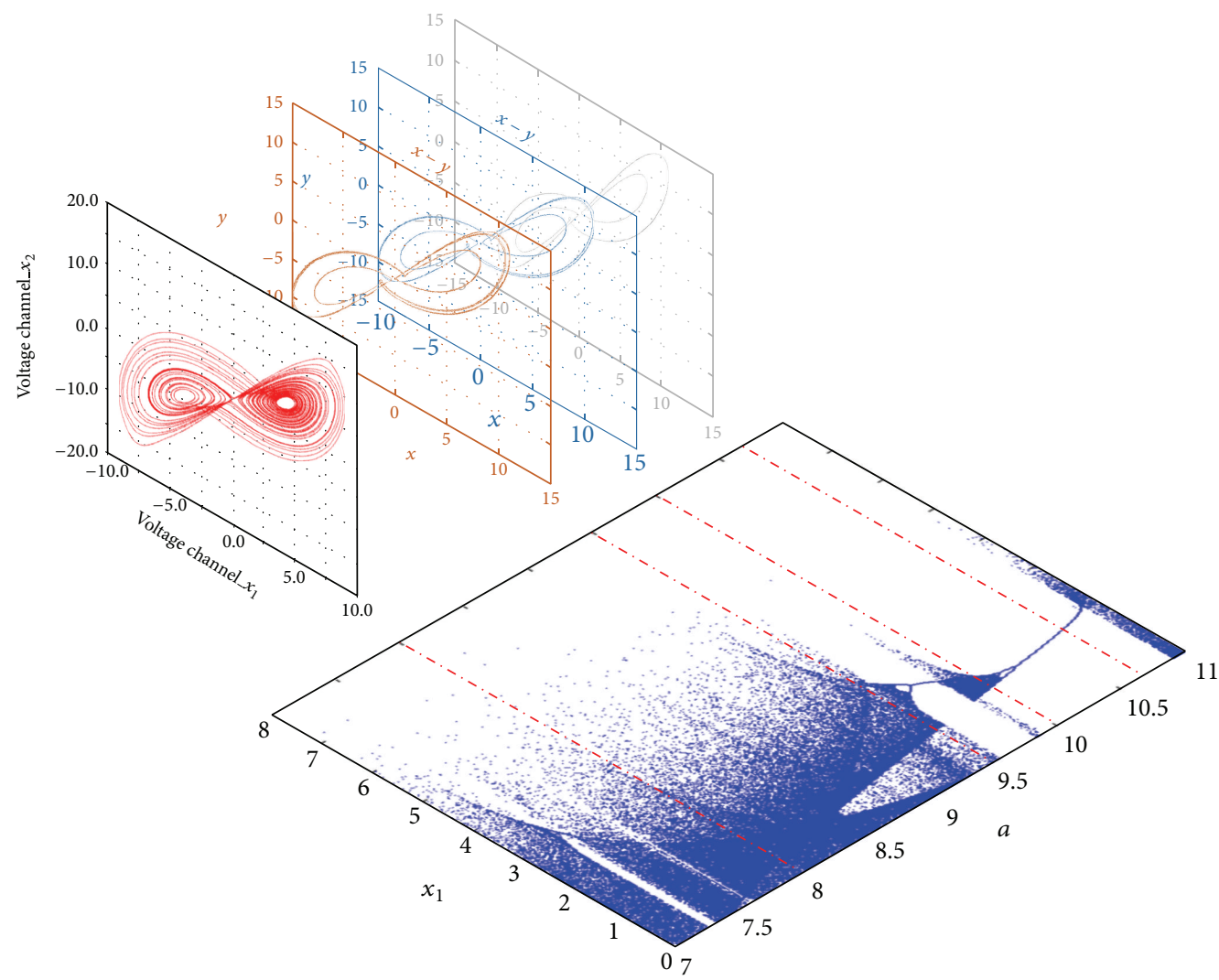

FIGURE 10: Bifurcation diagram with phase portraits of the four-dimensional Lorenz-Stenflo system.

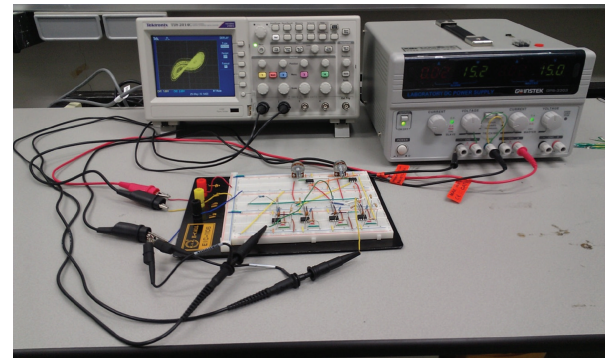

Figure 11: Experimental setup for realization of the fourdimensional Lorenz-Stenflo system.

where $\widetilde{A}_{j}=A_{j}(t)-\widehat{A}_{j}(t)+K p_{i},(j=1,2, \ldots, m)$. The $K p_{i}$ are positive constants which make the update laws always happen in the first quadrant.

Its derivative along the solution of (20) is

$$
\dot{V}(e, \widetilde{A})=f(t, x, A(t))-f(t, y, \widehat{A}(t))+\dot{F}(t)-u(t)+\dot{\widetilde{A}},
$$

where $u(t)$ and $\dot{\bar{A}}$ are chosen so that $\dot{V}=C e+D \widetilde{A}, C$ and $D$ are negative constants, and $\dot{V}$ is a negative definite function of $e_{1}, e_{2}, \ldots, e_{n}$ and $\widetilde{A}_{1}, \widetilde{A}_{2}, \ldots, \widetilde{A}_{m}$. When

$$
\lim _{t \rightarrow \infty} e=0, \quad \lim _{t \rightarrow \infty} \widetilde{A}=0,
$$

the generalized synchronization is obtained.
By using the GYC partial region stability theory, the Lyapunov function is easier to find, since the terms of first degree can be used to construct the definite Lyapunov function and the controller can be designed in lower order.

\section{Numerical Results of Adaptive Generalized Synchronization of the Four-Dimensional Lorenz-Stenflo System with Uncertain Chaotic Parameters via GYC Partial Region Stability Theory}

The master system is

$$
\begin{aligned}
& \dot{x}_{1}=A_{1}(t)\left(x_{2}-x_{1}\right) \\
& \dot{x}_{2}=A_{3}(t) x_{1}-x_{1} x_{3}-x_{2} \\
& \dot{x}_{3}=A_{2}(t) x_{3}-A_{4}(t) x_{3}+x_{1} x_{2} \\
& \dot{x}_{4}=-A_{1}(t) x_{4}-x_{1},
\end{aligned}
$$

where $A_{1}(t), A_{2}(t), A_{3}(t)$, and $A_{4}(t)$ are uncertain chaotic parameters. Equation (23) is called four-dimensional LorenzStenflo system with uncertain chaotic parameters. 

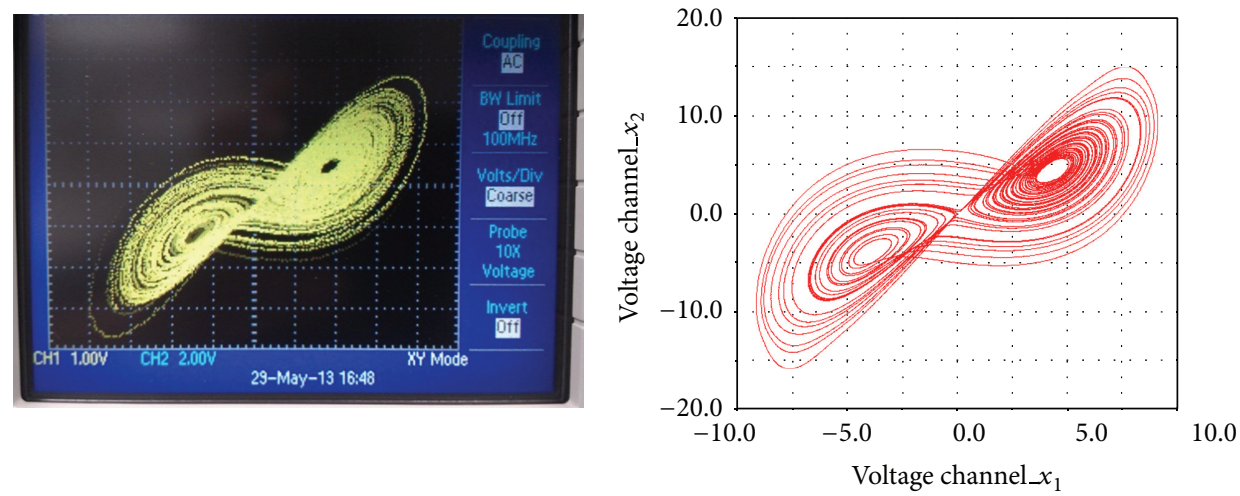

(a)
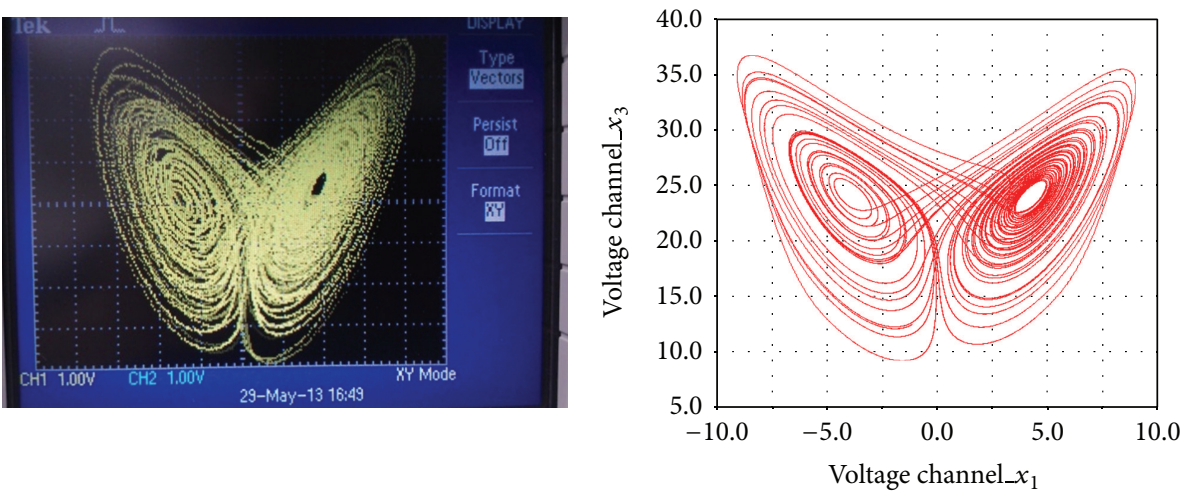

(b)
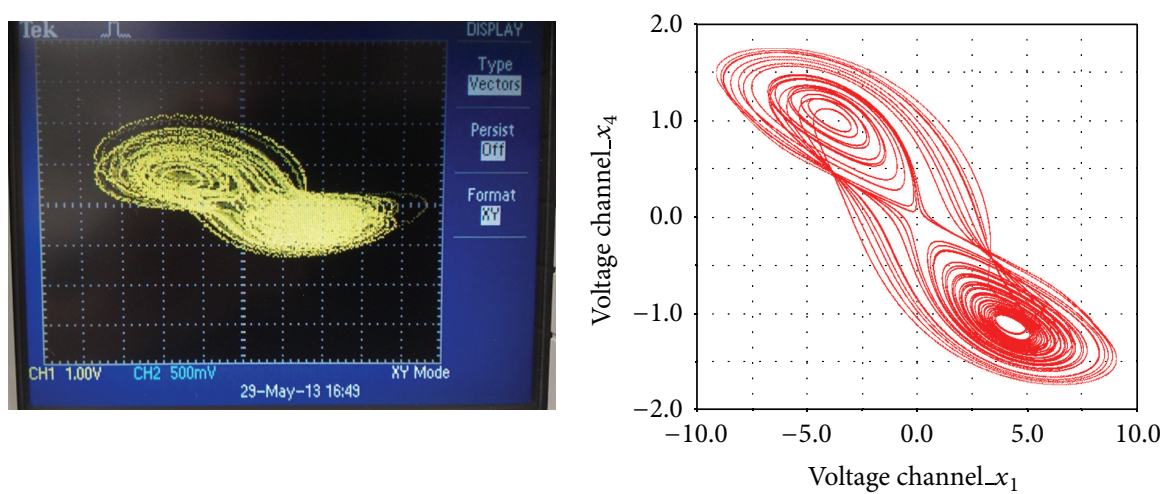

(c)
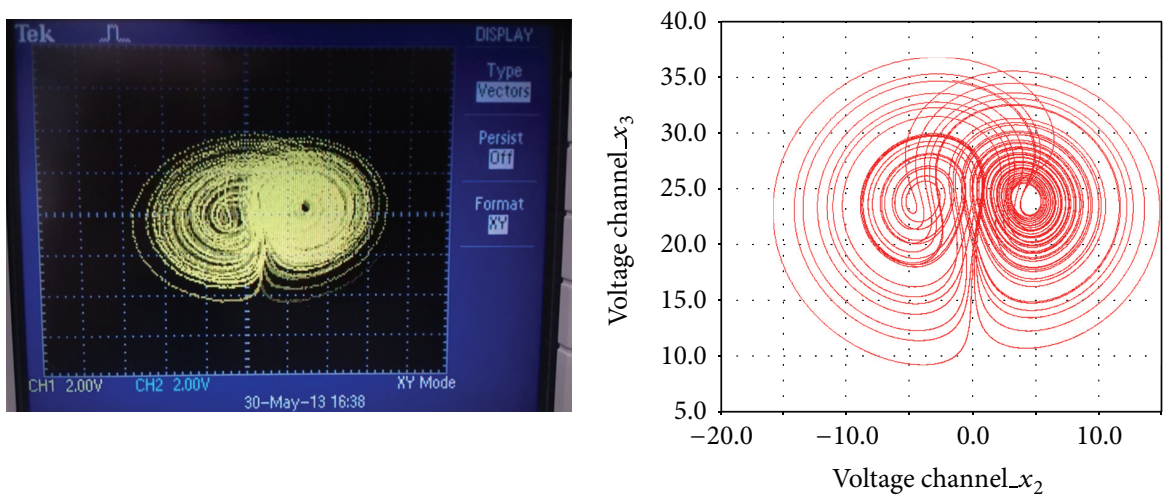

(d)

FIgUre 12: Continued. 

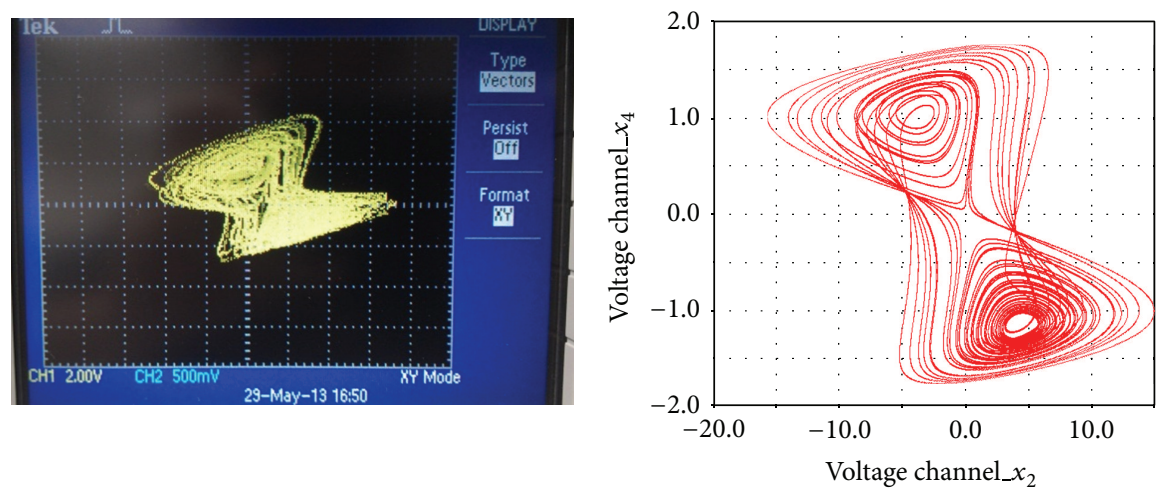

(e)
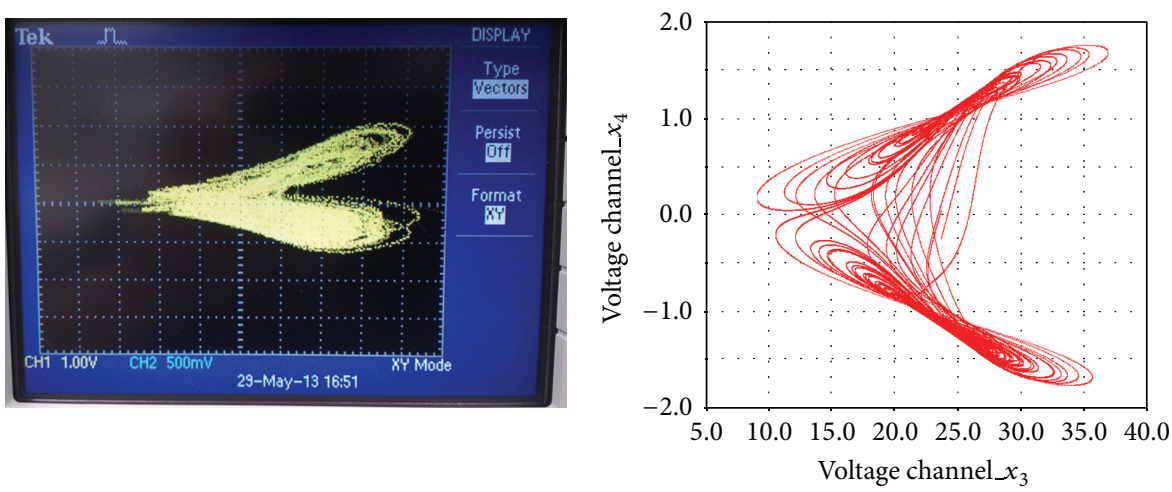

(f)

FIGURE 12: Realization and simulation circuit compare results for voltage signals outputs of the four-dimensional Lorenz-Stenflo system: (a) $V_{x_{1}}-V_{x_{2}}$, (b) $V_{x_{1}}-V_{x_{3}}$, (c) $V_{x_{1}}-V_{x_{4}}$, (d) $V_{x_{2}}-V_{x_{3}}$, (e) $V_{x_{2}}-V_{x_{4}}$, and (f) $V_{x_{3}}-V_{x_{4}}$.

Consider

$$
\begin{aligned}
& A_{1}(t)=a\left(1+f_{1} z_{1}\right) \\
& A_{2}(t)=b\left(1+f_{2} z_{2}\right) \\
& A_{3}(t)=c\left(1+f_{3} z_{3}\right) \\
& A_{4}(t)=d\left(1+f_{4} z_{4}\right),
\end{aligned}
$$

where $f_{1}, f_{2}, f_{3}$, and $f_{4}$ are arbitrary positive constant and $f_{1}=f_{2}=f_{3}=f_{4}=0.005$.

The chaotic signals system is

$$
\begin{aligned}
& \dot{z}_{1}=-z_{2} z_{3}+a z_{1}+b z_{4} \\
& \dot{z}_{2}=z_{1} z_{3}+c z_{2} \\
& \dot{z}_{3}=\frac{1}{3} z_{1} z_{2}+d z_{3} \\
& \dot{z}_{4}=z_{2} z_{3}+r z_{4},
\end{aligned}
$$

where $a=3.7, b=1.5, c=26$, and $d=0.7$.
The slave system is

$$
\begin{aligned}
& \dot{y}_{1}=\widehat{A}_{1}\left(y_{2}-y_{1}\right) \\
& \dot{y}_{2}=\widehat{A}_{3} y_{1}-y_{1} y_{3}-y_{2} \\
& \dot{y}_{3}=\widehat{A}_{2} y_{4}-\widehat{A}_{4} y_{3}+y_{1} y_{2} \\
& \dot{y}_{4}=-\widehat{A}_{1} y_{4}-y_{1},
\end{aligned}
$$

where $\widehat{A}_{1}(t), \widehat{A}_{2}(t), \widehat{A}_{3}(t)$, and $\widehat{A}_{4}(t)$ are estimates of uncertain chaotic parameters, respectively.

The nonlinear controllers, $u_{1}, u_{2}, u_{3}$, and $u_{4}$, are added to each equation in (26), respectively.

Consider

$$
\begin{aligned}
& \dot{y}_{1}=\widehat{A}_{1}\left(y_{2}-y_{1}\right)+u_{1} \\
& \dot{y}_{2}=\widehat{A}_{3} y_{1}-y_{1} y_{3}-y_{2}+u_{2} \\
& \dot{y}_{3}=\widehat{A}_{2} y_{4}-\widehat{A}_{4} y_{3}+y_{1} y_{2}+u_{3} \\
& \dot{y}_{4}=-\widehat{A}_{1} y_{4}-y_{1}+u_{4} .
\end{aligned}
$$

The given functional system for generalized synchronization is also the four-dimensional Lorenz-Stenflo system but with different initial conditions. 


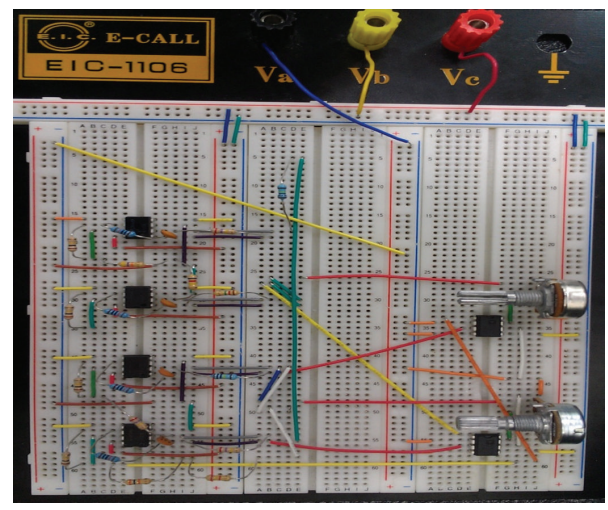

FIGURE 13: Realization circuit of the four-dimensional LorenzStenflo system.

TABLE 2: List of apparatus and components in the circuit experiment.

\begin{tabular}{lcc}
\hline Apparatus/Component & Model & Quantity \\
\hline Multiplier & $\mathrm{AD} 633$ & 2 \\
OP-Amplifier & $\mathrm{LF} 412$ & 4 \\
Capacitor & $2000 \mathrm{pF} \sim 0.47 \mathrm{uF}$ & 4 \\
& $1 \mathrm{k} \sim 1.2 \mathrm{M}$ & 19 \\
& $270 \mathrm{k} \Omega$ & 3 \\
& $38.461 \mathrm{k} \Omega$ & 1 \\
Resister & $667 \mathrm{k} \Omega$ & 1 \\
& $1429 \mathrm{k} \Omega$ & 1 \\
& $100 \mathrm{k} \Omega$ & 8 \\
Variable resistor & $1 \mathrm{M} \Omega$ & 4 \\
DC power supply & $100 \mathrm{k}$ & 2 \\
\hline
\end{tabular}

Consider

$$
\begin{aligned}
& \dot{w}_{1}=a\left(w_{2}-w_{1}\right) \\
& \dot{w}_{2}=c w_{1}-w_{1} w_{3}-w_{2} \\
& \dot{w}_{3}=b w_{4}-d w_{3}+w_{1} w_{2} \\
& \dot{w}_{4}=-a w_{4}-w_{1} .
\end{aligned}
$$

The initial value of the states of the master system, of the slave system, of the chaotic signals system, and of the given functional system are taken as $x_{1}(0)=x_{2}(0)=x_{3}(0)=$ $x_{4}(0)=0.2, y_{1}(0)=y_{2}(0)=y_{3}(0)=y_{4}(0)=0.3$, $z_{1}(0)=z_{2}(0)=z_{3}(0)=z_{4}(0)=0.7$, and $w_{1}(0)=w_{2}(0)=$ $w_{3}(0)=w_{4}(0)=0.5$, respectively.

The generalized synchronization error functions are

$$
e_{i}=x_{i}-y_{i}+w_{i}+K e_{i}, \quad(i=1,2,3,4)
$$

The addition of constant $K e_{i}$ makes the error states always greater than zero. As Figure 15 shows, we can confirm that
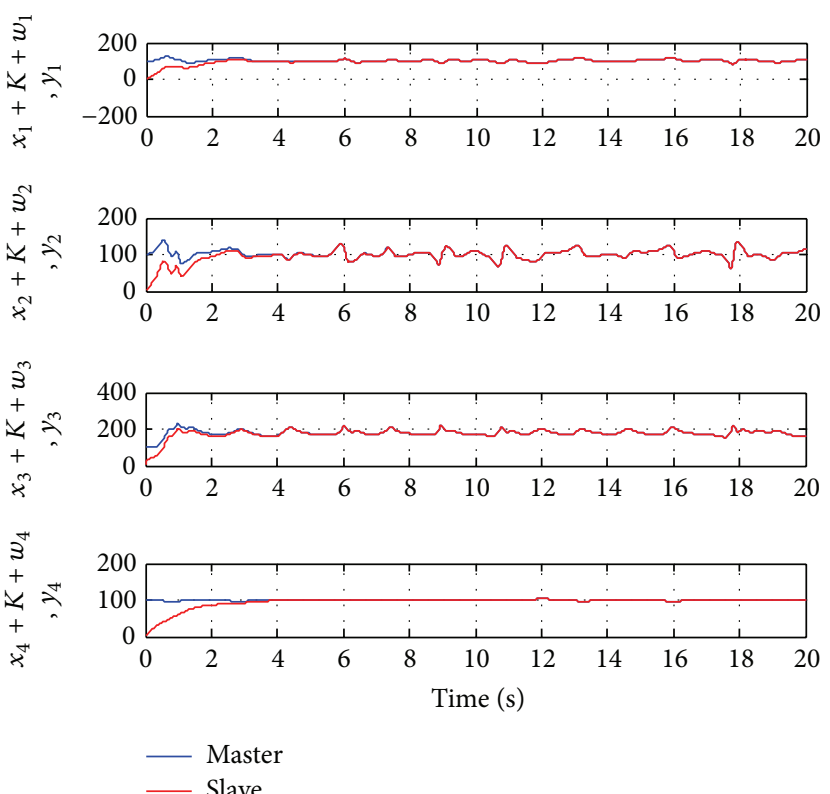

FIGURE 14: Time histories of the master system and slave system.

the error states are always greater than zero. From (29), we get the following error dynamics:

$$
\begin{aligned}
\dot{e}_{1}= & \widehat{A}_{1}\left(e_{2}-e_{1}-w_{2}+w_{1}\right) \\
& +\widetilde{A}_{1}\left(x_{2}-x_{1}\right)+a\left(w_{2}-w_{1}\right)-u_{1} \\
\dot{e}_{2}= & \widehat{A}_{3}\left(e_{1}-w_{1}-K\right)+\widetilde{A}_{3} x_{1} \\
& +c w_{1}-x_{1} x_{3}-w_{1} w_{3}+\left(x_{1}+w_{1}-e_{1}+K\right) \\
& \times\left(x_{3}+w_{3}-e_{3}+K\right)-\left(e_{2}-K\right)-u_{2} \\
\dot{e}_{3}= & \widehat{A}_{2}\left(e_{4}-w_{4}-K\right)+\widetilde{A}_{2} x_{4}+b w_{4} \\
& -\widehat{A}_{4}\left(e_{3}-w_{3}-K\right)-\widetilde{A}_{4} x_{3}-d w_{3} \\
& +x_{1} x_{3}+w_{1} w_{3}-\left(x_{1}+w_{1}-e_{1}+K\right) \\
& \times\left(x_{2}+w_{2}-e_{2}+K\right)-u_{3} \\
\dot{e}_{4}= & -\widehat{A}_{1}\left(e_{4}-w_{4}-K\right)-\widetilde{A}_{1} x_{4}-a w_{4}-\left(e_{1}-K\right)-u_{4},
\end{aligned}
$$

where $e_{1}=x_{1}-y_{1}+w_{1}, e_{2}=x_{2}-y_{2}+w_{2}, e_{3}=x_{3}-y_{3}+$ $w_{3}, e_{4}=x_{4}-y_{4}+w_{4}, \widetilde{A}_{1}=A_{1}-\widehat{A}_{1}+K p_{1}, \widetilde{A}_{2}=A_{2}-\widehat{A}_{2}+$ $K p_{2}, \widetilde{A}_{3}=A_{3}-\widehat{A}_{3}+K p_{3}$, and $\widetilde{A}_{4}=A_{4}-\widehat{A}_{4}+K p_{4}$. The addition of constant $K p_{i}(i=1,2,3,4)$ makes the update laws always greater than zero. As Figure 17 shows, we can confirm that the update laws are always greater than zero.

Choose a Lyapunov function in the form of a positive definite function:

$$
\begin{aligned}
V & \left(e_{1}, e_{2}, e_{3}, e_{4}, \widetilde{A}_{1}, \widetilde{A}_{2}, \widetilde{A}_{3}, \widetilde{A}_{4}\right) \\
& =\left(e_{1}+e_{2}+e_{3}+e_{4}+\widetilde{A}_{1}+\widetilde{A}_{2}+\widetilde{A}_{3}+\widetilde{A}_{4}\right)
\end{aligned}
$$



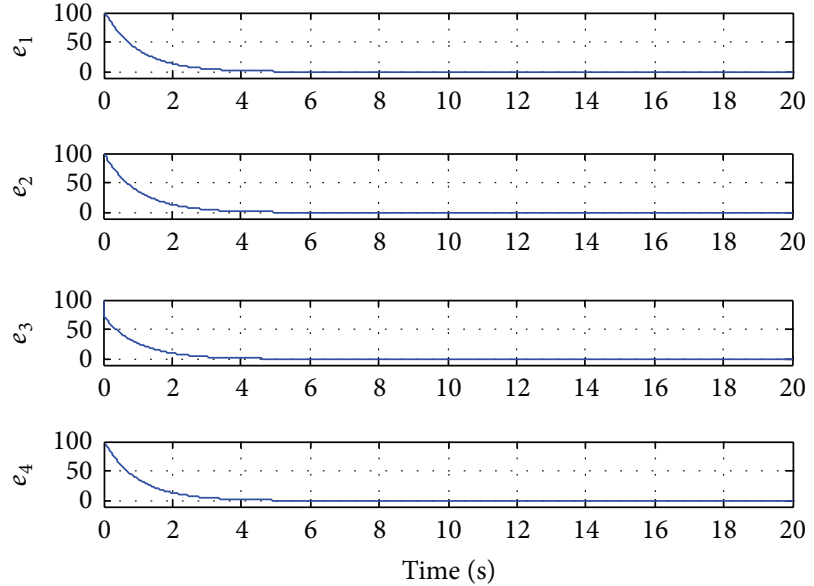

FIGURE 15: Time histories of error states.

and its time derivative is

$$
\begin{aligned}
\dot{V}\left(e_{1}, e_{2}, e_{3}, e_{4}, \widetilde{A}_{1}, \widetilde{A}_{2}, \widetilde{A}_{3}, \widetilde{A}_{4}\right) & \\
= & \dot{e}_{1}+\dot{e}_{2}+\dot{e}_{3}+\dot{e}_{4}+\dot{\tilde{a}}+\dot{\tilde{b}}+\dot{\tilde{c}}+\dot{\tilde{d}} \\
= & \left(\widehat{A}_{1}\left(e_{2}-e_{1}-w_{2}+w_{1}\right)+\widetilde{A}_{1}\left(x_{2}-x_{1}\right)\right. \\
& \left.+a\left(w_{2}-w_{1}\right)-u_{1}\right) \\
+ & \left(\widehat{A}_{3}\left(e_{1}-w_{1}-K\right)+\widetilde{A}_{3} x_{1}+c w_{1}-x_{1} x_{3}-w_{1} w_{3}\right. \\
& \quad+\left(x_{1}+w_{1}-e_{1}+K\right)\left(x_{3}+w_{3}-e_{3}+K\right) \\
& \left.\quad-\left(e_{2}-K\right)-u_{2}\right) \\
+ & \left(\widehat{A}_{2}\left(e_{4}-w_{4}-K\right)+\widetilde{A}_{2} x_{4}+b w_{4}-\widehat{A}_{4}\left(e_{3}-w_{3}-K\right)\right. \\
& \quad-\widetilde{A}_{4} x_{3}-d w_{3}+x_{1} x_{3}+w_{1} w_{3} \\
& \left.\quad-\left(x_{1}+w_{1}-e_{1}+K\right)\left(x_{2}+w_{2}-e_{2}+K\right)-u_{3}\right) \\
+ & \left(-\widehat{A}_{1}\left(e_{4}-w_{4}-K\right)-\widetilde{A}_{1} x_{4}-a w_{4}-\left(e_{1}-K\right)-u_{4}\right) \\
+ & (-\dot{\hat{a}})+(-\dot{\vec{b}})+(-\dot{\vec{c}})+(-\dot{\vec{d}})+(-\dot{\vec{r}}) .
\end{aligned}
$$
ters as

We choose the update laws for those uncertain parame-

$$
\begin{aligned}
& \dot{\widetilde{A}}_{1}=\dot{A}_{1}-\dot{\widehat{A}}_{1}=-\widetilde{A}_{1} e_{1}-\widetilde{A}_{1} \\
& \dot{\widetilde{A}}_{2}=\dot{A}_{2}-\dot{\widehat{A}}_{2}=-\widetilde{A}_{2} e_{2}-\widetilde{A}_{2} \\
& \dot{\vec{A}}_{3}=\dot{A}_{3}-\dot{A}_{3}=-\widetilde{A}_{3} e_{3}-\widetilde{A}_{3} \\
& \dot{\vec{A}}_{4}=\dot{A}_{4}-\dot{\widehat{A}}_{4}=-\widetilde{A}_{4} e_{4}-\widetilde{A}_{4} .
\end{aligned}
$$

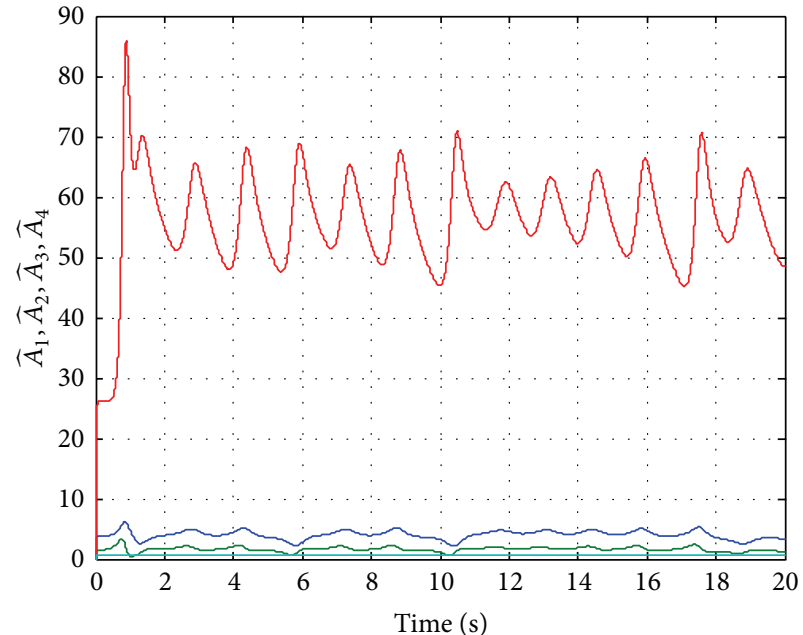

$\begin{array}{ll}\widehat{A}_{1} & -\widehat{A}_{3} \\ \widehat{A}_{2} & -\widehat{A}_{4}\end{array}$

FIgURE 16: Time histories of estimated parameters.

The initial values of estimates for uncertain parameters are $\widehat{A}_{1}(0)=\widehat{A}_{2}(0)=\widehat{A}_{3}(0)=\widehat{A}_{4}(0)=0$. Through (32) and (33), the appropriate controllers can be designed as

$$
\begin{aligned}
u_{1}= & \widehat{A}_{1}\left(e_{2}-e_{1}-w_{2}+w_{1}\right) \\
& +\widetilde{A}_{1}\left(x_{2}-x_{1}-x_{4}-e_{1}\right)+a\left(w_{2}-w_{1}\right)+e_{1} \\
u_{2}= & \widehat{A}_{3}\left(e_{1}-w_{1}-K\right)+\widetilde{A}_{2}\left(x_{4}-e_{2}\right) \\
& +c w_{1}-x_{1} x_{3}-w_{1} w_{3} \\
& +\left(x_{1}-e_{1}+w_{1}+K\right)\left(x_{3}-e_{3}+w_{3}+K\right)+e_{2} \\
u_{3}= & \widehat{A}_{2}\left(e_{4}-w_{4}-K\right)+b w_{4}-\widehat{A}_{4}\left(e_{3}-w_{3}-K\right) \\
& -d w_{3}+\widetilde{A}_{3}\left(x_{1}-e_{3}\right)+x_{1} x_{2}+w_{1} w_{2} \\
& -\left(x_{1}-e_{1}+w_{1}+K\right)\left(x_{2}-e_{2}+w_{2}+K\right)+e_{3} \\
u_{4}= & -\widehat{A}_{1}\left(e_{4}-w_{4}-K\right)-a w_{4} \\
& +\widetilde{A}_{4}\left(-x_{3}-e_{4}\right)-\left(e_{1}-K\right)+e_{4} .
\end{aligned}
$$

Substituting (33) and (34) into (32), we obtain

$$
\dot{V}=-e_{1}-e_{2}-e_{3}-e_{4}-\widetilde{A}_{1}-\widetilde{A}_{2}-\widetilde{A}_{3}-\widetilde{A}_{4}<0
$$

which is a negative-definite function of $e_{1}, e_{2}, e_{3}, e_{4}, \widetilde{A}_{1}$, $\widetilde{A}_{2}, \widetilde{A}_{3}$, and $\widetilde{A}_{4}$. The numerical simulation results are shown in Figures 14, 15, 16, and 17.

\section{Conclusions}

In summary, the paper has studied the nonlinear dynamical behaviors of the new chaotic attractor, including some basic dynamical properties, bifurcations, Lyapunov exponents, Lyapunov dimension, periodic motion, and routes to chaos 


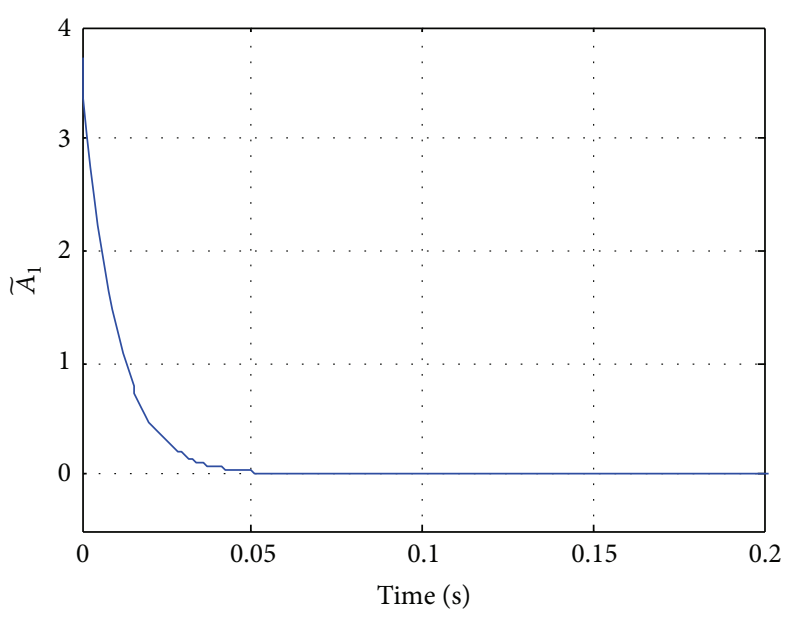

(a)

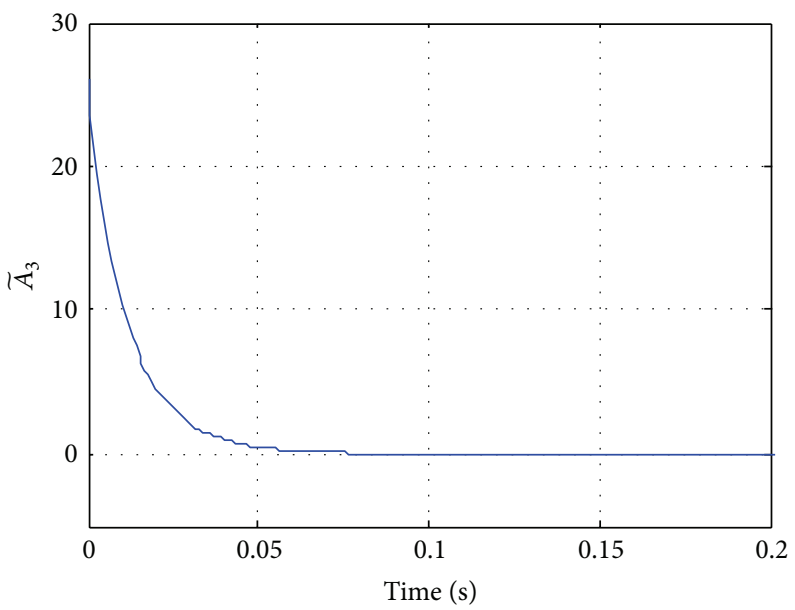

(c)

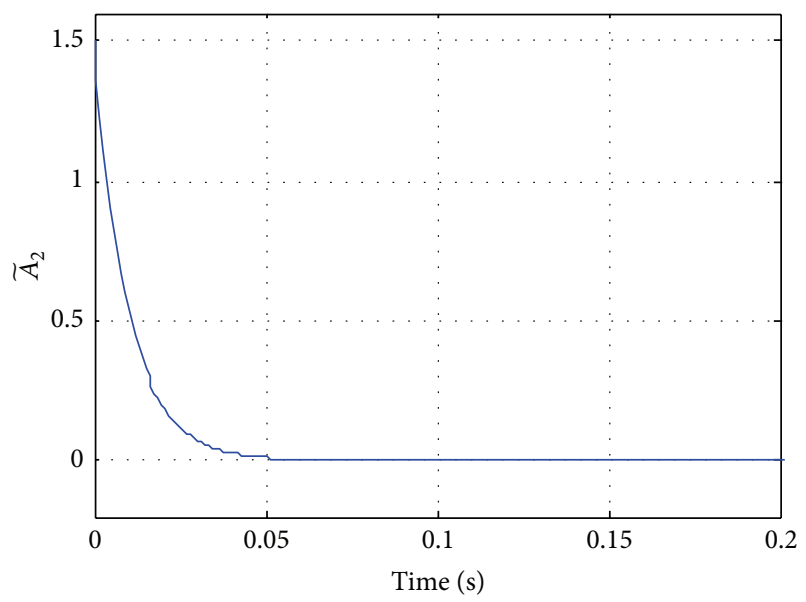

(b)

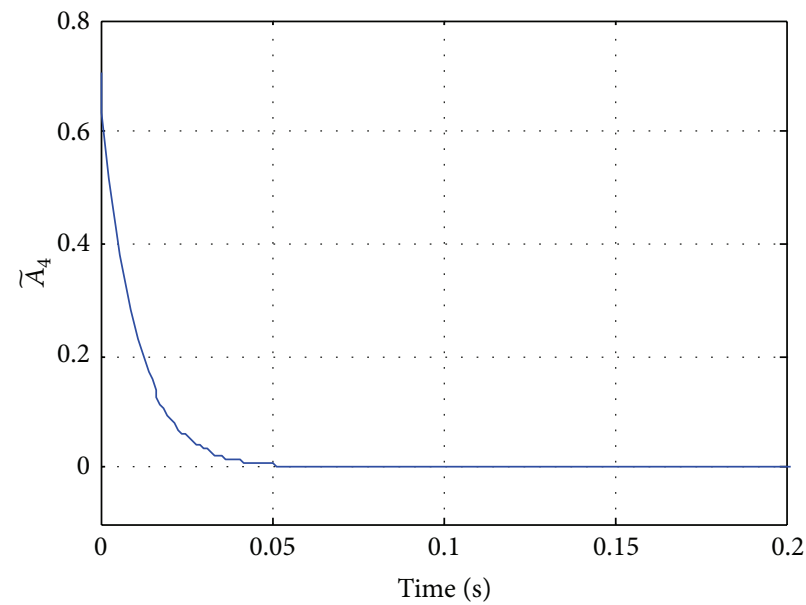

(d)

FIGURE 17: Time histories of parameters differences.

motion. Also, the four-dimensional Lorenz-Stenflo system based realization circuit has been constructed to implement various wing butterfly attractors. Furthermore, by electronic circuit implementation of the proposed four-dimensional Lorenz-Stenflo system, it is shown that the chaotic attractors do physically exist. A new strategy, the GYC partial region stability theory, is proposed to achieve adaptive chaos synchronization with uncertain chaotic parameters.

\section{Conflict of Interests}

The authors declare that there is no conflict of interests regarding the publication of this paper.

\section{Acknowledgment}

This research was supported by the National Science Council, Republic of China, under Grant no. NSC 102-2221-E-011-034-

\section{References}

[1] Z. Li, Y. Soh, and C. Wen, Switched and Impulsive Systems, Analysis, Design, and Applications, vol. 313, Springer, Berlin, Germany, 2005.

[2] P. Galajda and D. Kocur, "Chua's circuit in spread spectrum communication systems," Radio Engineering, vol. 11, no. 2, pp. 6-10, 2002.

[3] L. Kocarev, Z. Galias, and S. Lian, Eds., Intelligent Computing Based on Chaos, vol. 184 of Studies in Computational Intelligence, Springer, Berlin, Germany, 2009.

[4] K. Grygiel and P. Szlachetka, "Lyapunov exponents analysis of autonomous and nonautonomous sets of ordinary differential equations," Acta Physica Polonica B, vol. 26, no. 8, pp. 1321-1331, 1995.

[5] S. H. Strogatz, Nonlinear Dynamics and Chaos: With Applications to Physics, Biology, Chemistry, and Engineering, Perseus Books, Cambridge, Mass, USA, 1994.

[6] K. Kiers, D. Schmidt, and J. C. Sprott, "Precision measurements of a simple chaotic circuit," American Journal of Physics, vol. 72, no. 4, pp. 503-509, 2004. 
[7] E. N. Lorenz, "Deterministic nonperiodic flow," Journal of the Atmospheric Sciences, vol. 20, pp. 130-141, 1963.

[8] L. Stenflo, "Generalized Lorenz equations for acoustic-gravity waves in the atmosphere," Physica Scripta, vol. 53, no. 1, pp. 8384, 1996.

[9] J. C. Xavier and P. C. Rech, "Regular and chaotic dynamics of the Lorenz-Stenflo system," International Journal of Bifurcation and Chaos in Applied Sciences and Engineering, vol. 20, no. 1, pp. 145-152, 2010.

[10] P. Wang, D. Li, and Q. Hu, "Bounds of the hyper-chaotic Lorenz-Stenflo system," Communications in Nonlinear Science and Numerical Simulation, vol. 15, no. 9, pp. 2514-2520, 2010.

[11] H. Li, X. Liao, and X. Lei, "Two fuzzy control schemes for Lorenz-Stenflo chaotic system," Journal of Vibration and Control, vol. 18, no. 11, pp. 1675-1682, 2012.

[12] W. Xiang, "Equilibrium points and bifurcation control for Lorenz-Stenflo system," ICIC Express Letters, vol. 3, no. 1, pp. 61-66, 2009.

[13] C.-H. Yang, "Chaos hybrid generalized synchronization of liuchen system by GYC partial region stability theory," Journal of Computational and Theoretical Nanoscience, vol. 10, no. 4, pp. 825-831, 2013.

[14] Z.-M. Ge and C.-H. Yang, "The generalized synchronization of a Quantum-CNN chaotic oscillator with different order systems," Chaos, Solitons and Fractals, vol. 35, no. 5, pp. 980-990, 2008.

[15] Z.-M. Ge, C.-W. Yao, and H.-K. Chen, "Stability on partial region in dynamics," Journal of the Chinese Society of Mechanical Engineers, vol. 15, no. 2, pp. 140-151, 1994.

[16] Z.-M. Ge, J.-K. Yu, and H.-K. Chen, "Three asymptotical stability theorems on partial region with applications," Japanse Journal of Applied Physics, vol. 37, article 2762, 1998.

[17] P. Frederickson, J.-L. Kaplan, E.-D. Yorke, and J.-A. Yorke, "The Liapunov dimension of strange attractors," Journal of Differential Equations, vol. 49, no. 2, pp. 185-207, 1983.

[18] X. Wang and G. A. Chen, "A chaotic system with only one stable equilibrium," Communications in Nonlinear Science and Numerical Simulation, vol. 17, no. 3, pp. 1264-1272, 2012.

[19] Y. Liu, "Circuit implementation and finite-time synchronization of the 4D Rabinovich hyperchaotic system," Nonlinear Dynamics, vol. 67, no. 1, pp. 89-96, 2012.

[20] A. G. Radwan, A. M. Soliman, and A. L. El-Sedeek, "MOS realization of the double-scroll-like chaotic equation'"' IEEE Transactions on Circuits and Systems I: Fundamental Theory and Applications, vol. 50, no. 2, pp. 285-288, 2003.

[21] A. Abooee, H. A. Yaghini-Bonabi, and M. R. Jahed-Motlagh, "Analysis and circuitry realization of a novel three-dimensional chaotic system," Communications in Nonlinear Science and Numerical Simulation, vol. 18, no. 5, pp. 1235-1245, 2013. 


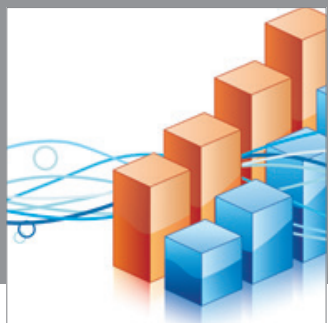

Advances in

Operations Research

mansans

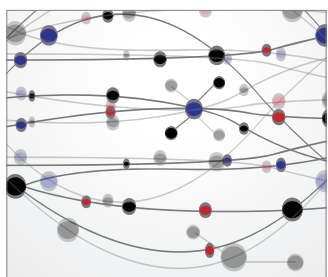

The Scientific World Journal
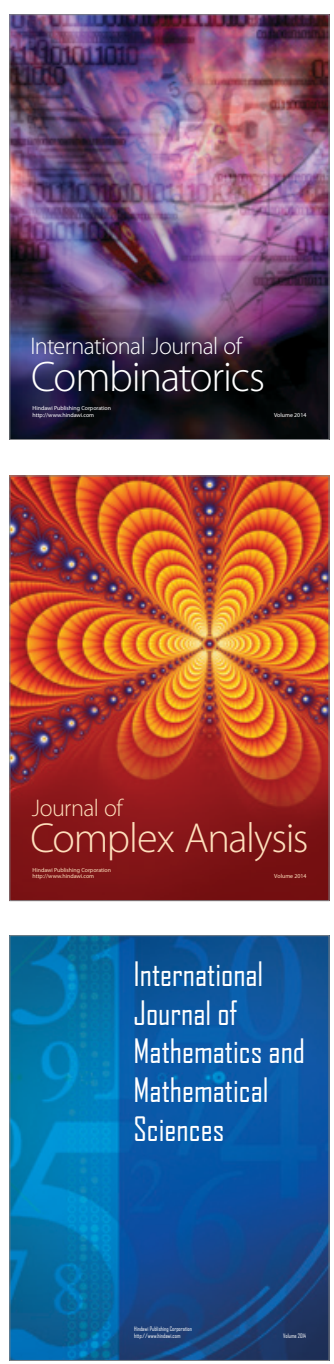
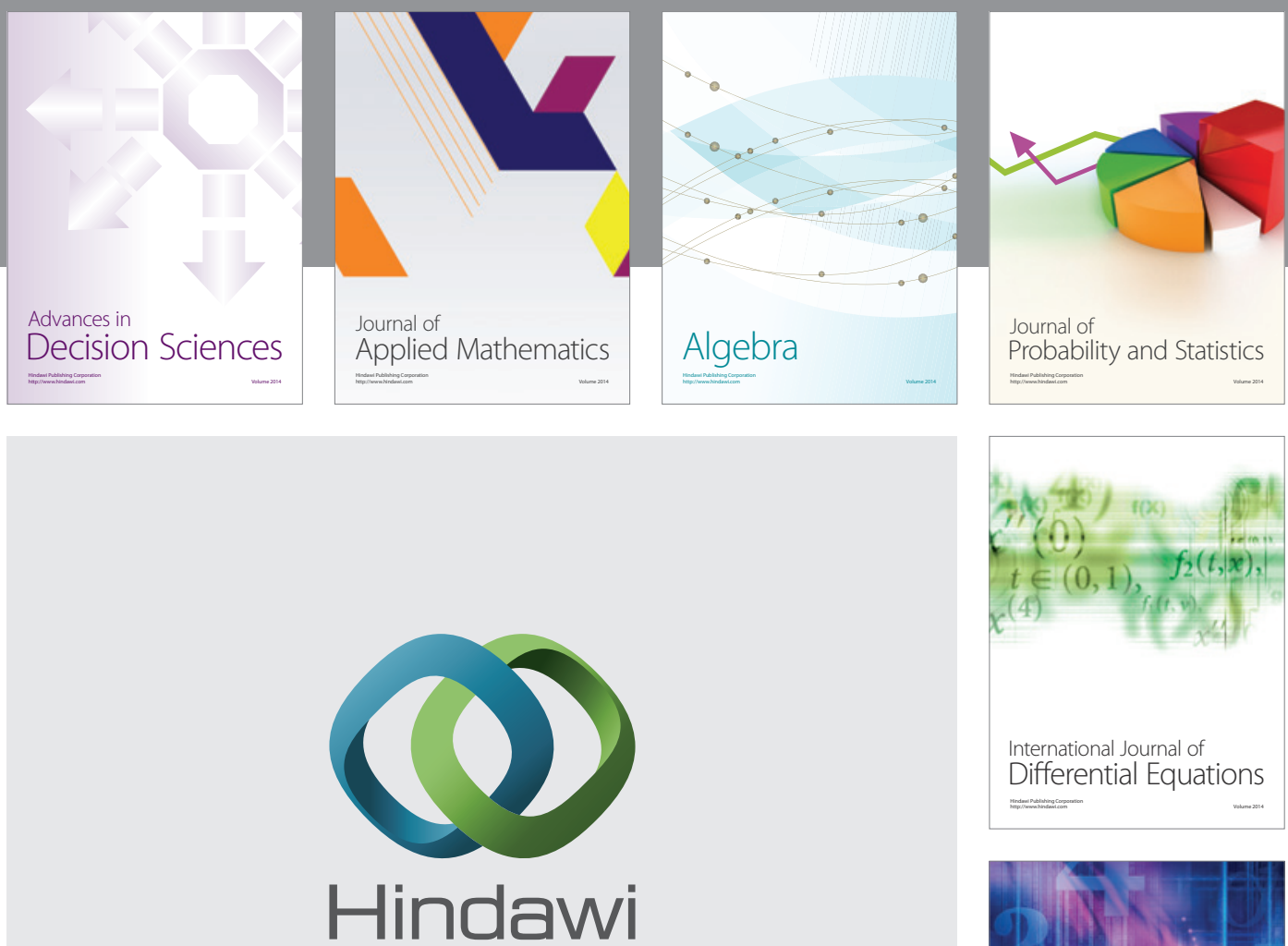

Submit your manuscripts at http://www.hindawi.com
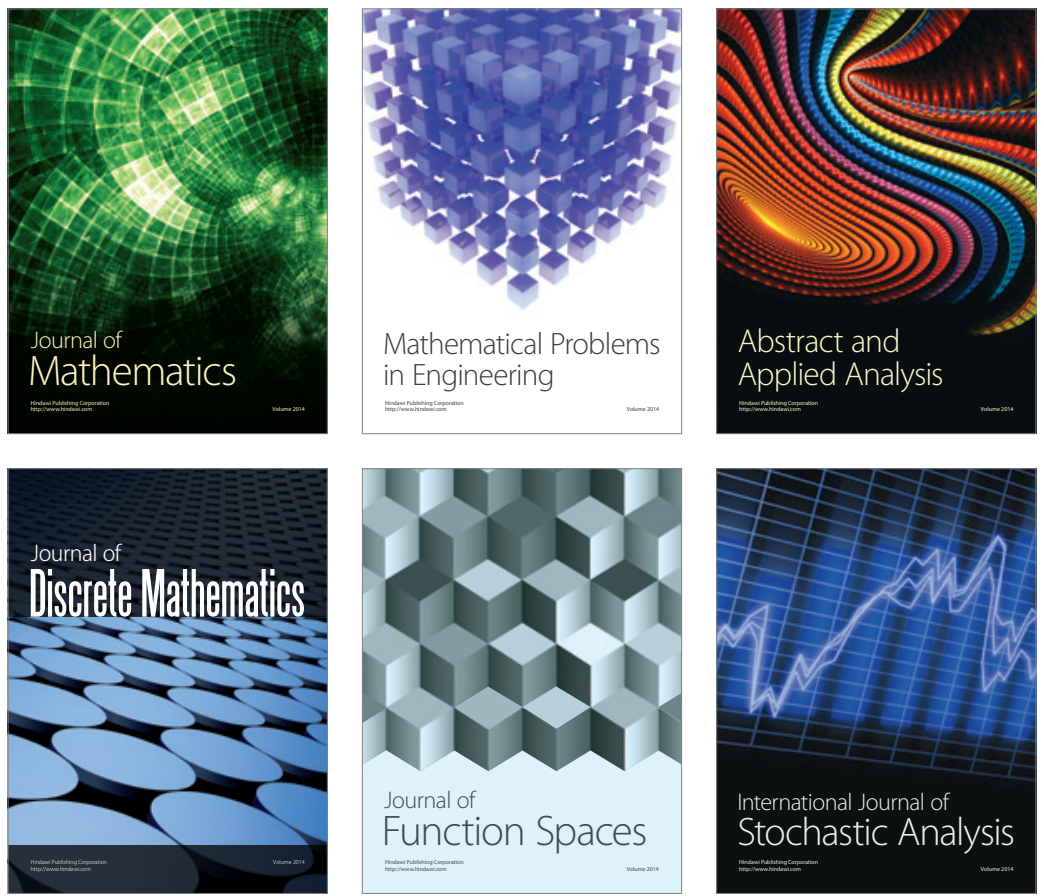

Journal of

Function Spaces

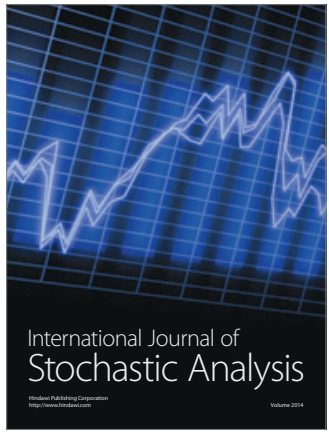

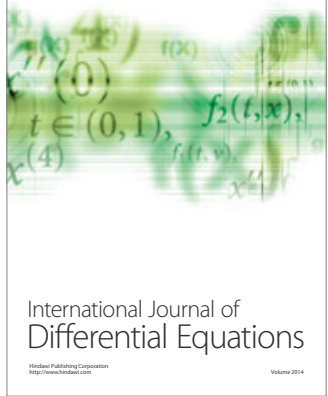
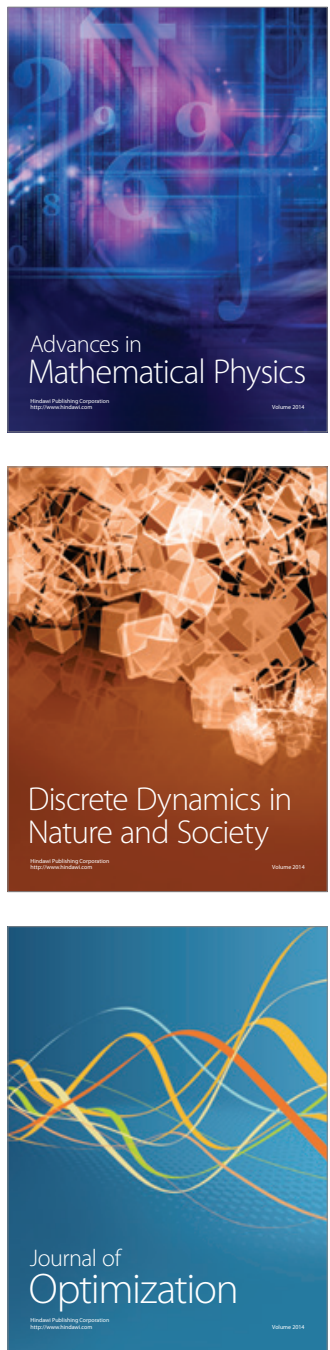